TRANSACTIONS OF THE

AMERICAN MATHEMATICAL SOCIETY

Volume 352, Number 1, Pages 285-310

S 0002-9947(99)02528-3

Article electronically published on September 21, 1999

\title{
ABSTRACT PARABOLIC PROBLEMS WITH CRITICAL NONLINEARITIES AND APPLICATIONS TO NAVIER-STOKES AND HEAT EQUATIONS
}

\author{
JOSÉ M. ARRIETA AND ALEXANDRE N. CARVALHO
}

\begin{abstract}
We prove a local existence and uniqueness theorem for abstract parabolic problems of the type $\dot{x}=A x+f(t, x)$ when the nonlinearity $f$ satisfies certain critical conditions. We apply this abstract result to the Navier-Stokes and heat equations.
\end{abstract}

\section{INTRODUCTION}

In this paper we consider problems of the type

$$
\begin{gathered}
\dot{x}=A x+f(t, x), \quad t>t_{0}, \\
x\left(t_{0}\right)=x_{0},
\end{gathered}
$$

where the linear operator $A: D(A) \subset X^{0} \rightarrow X^{0}$ satisfies that $-A$ is a sectorial operator in the Banach space $X^{0}$. We will denote by $X^{\alpha}, \alpha \geq 0$, the fractional power spaces associated to the operator $A$ (see [HE, AM1, AM2, AM3]) and by $e^{A t}$ the analytic semigroup generated by $A$. Without loss of generality we can assume that $e^{A t}$ is uniformly bounded, that is,

$$
\begin{aligned}
& \left\|e^{A t} x\right\|_{X^{\alpha}} \leq M\|x\|_{X^{\alpha}}, \quad \alpha \geq 0, \\
& \left\|e^{A t} x\right\|_{X^{\alpha}} \leq M t^{-\alpha}\|x\|_{X^{0}}, \quad \alpha \geq 0 .
\end{aligned}
$$

In order to initiate the discussion let us assume for a moment that the map $f$ is time independent and $t_{0}=0$. Therefore the problem above reads

$$
\begin{gathered}
\dot{x}=A x+f(x), \quad t>0, \\
x(0)=x_{0} .
\end{gathered}
$$

It is well known now that if the map $f: X^{1} \rightarrow X^{\alpha}$, for some $\alpha>0$, and is Lipschitz on bounded sets of $X^{1}$, that is, $\|f(x)-f(y)\|_{X^{\alpha}} \leq C(R)\|x-y\|_{X^{1}}$, for $\|x\|_{X^{1}},\|y\|_{X^{1}} \leq R$, then the problem (3) is locally well posed in $X^{1}$. For each $x_{0} \in X^{1}$ one seeks fixed points of the map $T$ in the space $K(\tau, \mu)=\{x(t) \in$

Received by the editors August 6, 1997.

1991 Mathematics Subject Classification. Primary 34G20, 58D25; Secondary 35K05, 35Q30.

Key words and phrases. Abstract parabolic equations, critical nonlinearities, growth conditions, local existence, uniqueness, Navier-Stokes, heat equations.

The first author's research was partially supported by FAPESP-SP-Brazil, grant \# 1996/32894. The second author's research was partially supported by CNPq-Brazil, grant \# 300.889/92-5. 
$\left.C\left([0, \tau], X^{1}\right) ; x(0)=x_{0},\|x(t)\|_{L^{\infty}\left(0, \tau, X^{1}\right)} \leq\left\|x_{0}\right\|_{X^{1}}+\mu\right\}$, where $T$ is given by

$$
(T x)(t)=e^{A t} x_{0}+\int_{0}^{t} e^{A(t-s)} f(x(s)) d s .
$$

The simple computations,

$$
\begin{aligned}
& \|(T x)(t)-(T y)(t)\|_{X^{1}} \leq M \int_{0}^{t}(t-s)^{-1+\alpha}\|f(x(s))-f(y(s))\|_{X^{\alpha}} d s \\
& \leq C M \int_{0}^{t}(t-s)^{-1+\alpha}\|x(s)-y(s)\|_{X^{1}} d s \\
& \leq\left(C M \int_{0}^{t}(t-s)^{-1+\alpha} d s\right) \sup _{0 \leq s \leq t}\left\{\|x(s)-y(s)\|_{X^{1}}\right\}
\end{aligned}
$$

and

$$
\begin{gathered}
\|(T x)(t)\|_{X^{1}} \leq\left\|e^{A t} x_{0}\right\|_{X^{1}}+M \int_{0}^{t}(t-s)^{-1+\alpha}\|f(x(s))\|_{X^{\alpha}} d s \\
\leq\left\|e^{A t} x_{0}\right\|_{X^{1}}+C M \int_{0}^{t}(t-s)^{-1+\alpha} d s \\
+\left(C M \int_{0}^{t}(t-s)^{-1+\alpha} d s\right) \sup _{0 \leq s \leq t}\left\{\|x(s)\|_{X^{1}}\right\}
\end{gathered}
$$

together with the fact that $\left\|e^{A t} x_{0}\right\|_{X^{1}} \rightarrow\left\|x_{0}\right\|_{X^{1}}$ and $\int_{0}^{t}(t-s)^{-1+\alpha} d s=$ $t^{\alpha} \int_{0}^{1}(1-s)^{-1+\alpha} d s \rightarrow 0$ as $t \rightarrow 0^{+}$, suggest that for $\mu>0$ fixed we can choose $\tau>0$ small enough so that $T: K(\tau, \mu) \rightarrow K(\tau, \mu)$ and $T$ is a strict contraction in $K(\tau, \mu)$. Once this is accomplished, the Banach fixed point theorem takes care of the existence and uniqueness of solutions of the integral equation. With some extra effort one can show that the solution found is a solution of (3).

In the analysis above, the convergence of the improper integral $\int_{0}^{1}(1-s)^{-1+\alpha} d s$, which is equivalent to the fact that $\alpha>0$, is essential, and the whole argument breaks down when $\alpha=0$. In other words, since $A: X^{1} \rightarrow X^{0}$, the fact that $f: X^{1} \rightarrow X^{\alpha}$ with $\alpha>0$ means that the solutions of problem (3) can be obtained as perturbations of the solutions of the linear problem $\dot{x}=A x$.

In this paper we address the question of local solvability of problem (1), (3) when $\alpha=0$.

It is clear that if the only requirement on $f$ is that $f: X^{1} \rightarrow X^{0}$ be locally Lipschitz, it will be impossible to show that problem (3) is well posed. For example, taking $f(x)=-2 A x$, which satisfies $f: X^{1} \rightarrow X^{0}$ and is globally Lipschitz, we will have $\dot{x}=A x+f(x)=-A x$, which is not locally well posed, in general (if $A=\Delta$ then $\dot{x}=-A x$ is the backwards heat equation). Hence, some extra conditions should be imposed on $f$ to guarantee the existence of solutions of the above problem.

In order to illustrate the main ideas and techniques of this paper, let us consider the particular example given by the equation

$$
\begin{aligned}
& u_{t}=\Delta u+u|u|^{\rho-1} \quad \text { in } \Omega, \\
& u=0 \text { on } \partial \Omega, \\
& u(0)=u_{0},
\end{aligned}
$$

where $\Omega$ is a bounded and smooth domain in $\mathbb{R}^{3}$ and $\rho>1$. 
It is well known that the operator $\Delta$ can be regarded as an unbounded operator in $X^{0}=H^{-1}(\Omega)$ with domain $X^{1}=H_{0}^{1}(\Omega)$. Moreover, the fractional power spaces are given by $X^{\alpha}$ which satisfy the following embedding properties:

$$
\begin{array}{ll}
X^{\alpha} \hookrightarrow H^{2 \alpha-1}(\Omega), & \alpha>1 / 2, \\
X^{\frac{1}{2}}=L^{2}(\Omega), & \\
X^{\alpha} \hookleftarrow H^{2 \alpha-1}(\Omega), &
\end{array}
$$

(see [HE, AM2]).

If $f(u)=u|u|^{\rho-1}$, then with some Sobolev embeddings and with (6), we can show that for $1<\rho \leq 3$ we have $f: X^{1} \equiv H_{0}^{1}(\Omega) \rightarrow X^{\frac{1}{2}} \equiv L^{2}$; for $4<\rho \leq 5$ we have $f: X^{1} \equiv H_{0}^{1}(\Omega) \rightarrow H^{\frac{3-\rho}{2}} \hookrightarrow X^{\frac{5-\rho}{4}}$. Hence, for $1<\rho<5, f: X^{1} \rightarrow X^{\alpha}$ for some $\alpha>0$. For $\rho=5, f: X^{1} \rightarrow X^{0}$ and we are in the critical case $\alpha=0$. But observe that for $\rho=5$, again with some Sobolev embeddings and (6), we get that if $\epsilon>0$ is small then $f: X^{1+\epsilon} \rightarrow X^{5 \epsilon}$, while the linear operator $A: X^{1+\epsilon} \rightarrow X^{\epsilon}$. This means that, although $A$ and $f$ can be regarded as of the same order in $X^{1}$, if we consider an slightly better space, $X^{1+\epsilon}$, then the map $f$ regularizes more than $A\left(X^{5 \epsilon}\right.$ is a better space than $\left.X^{\epsilon}\right)$. Moreover, it can be seen that $f$ satisfies

$$
\begin{gathered}
\|f(u)-f(v)\|_{X^{5 \epsilon}} \leq c\|u-v\|_{X^{1+\epsilon}}\left(\|u\|_{X^{1+\epsilon}}^{4}+\|v\|_{X^{1+\epsilon}}^{4}+1\right) \quad \forall u, v \in X^{1+\epsilon}, \\
\|f(u)\|_{X^{5 \epsilon}} \leq c\|u\|_{X^{1+\epsilon}}^{5} .
\end{gathered}
$$

In particular, this means that we can solve problem (5) with initial data in $X^{1+\epsilon}$.

Moreover, if we consider now a sequence of initial data $u_{n} \in X^{1+\epsilon}$ with $u_{n} \rightarrow$ $u_{0} \in X^{1}$ in $X^{1}$, with the computation

$$
\begin{aligned}
& t^{\epsilon}\left\|u_{n}(t)\right\|_{X^{1+\epsilon}} \leq t^{\epsilon}\left\|e^{A t} u_{n}\right\|_{X^{1+\epsilon}}+t^{\epsilon} \int_{0}^{t}(t-s)^{-1+4 \epsilon}\left\|u_{n}(s)^{5}\right\|_{X^{5 \epsilon}} d s \\
& \leq t^{\epsilon}\left\|e^{A t} u_{n}\right\|_{X^{1+\epsilon}}+t^{\epsilon} \int_{0}^{t}(t-s)^{-1+4 \epsilon} s^{-5 \epsilon} d s \sup _{0<s<t}\left\{s^{\epsilon}\left\|u_{n}(s)\right\|_{X^{1+\epsilon}}\right\}^{5}
\end{aligned}
$$

and the fact that $t^{\epsilon}\left\|e^{A t} u_{n}\right\|_{X^{1+\epsilon}} \stackrel{t \rightarrow 0^{+}}{\longrightarrow} 0$ uniformly on compacts of $X^{1}$ (see Lemma 2, below), it is not difficult to see that if $\mu>0$ is small enough, we can get a uniform time $\tau_{1}>0$, independent of $n$, such that $t^{\epsilon}\left\|u_{n}(t)\right\|_{X^{1+\epsilon}} \leq \mu$ for all $t \in\left(0, \tau_{1}\right]$ and all $n$.

But also, for $0<t \leq \tau_{1}$, we have

$$
\begin{aligned}
& t^{\epsilon}\left\|u_{n}(t)-u_{m}(t)\right\|_{X^{1+\epsilon}} \leq t^{\epsilon}\left\|e^{A t}\left(u_{n}-u_{m}\right)\right\|_{X^{1+\epsilon}} \\
& \quad+t^{\epsilon} \int_{0}^{t}(t-s)^{-1+4 \epsilon}\left\|u_{n}(s)-u_{m}(s)\right\|_{X^{1+\epsilon}}\left(1+\left\|u_{n}(s)\right\|_{X^{1+\epsilon}}^{4}+\left\|u_{m}(s)\right\|_{X^{1+\epsilon}}^{4}\right) d s \\
& \leq\left\|u_{n}-v_{n}\right\|_{X^{1}}+\left(t^{\epsilon} \int_{0}^{t}(t-s)^{-1+4 \epsilon} s^{-\epsilon} d s\right) \sup _{0<s \leq t}\left\{s^{\epsilon}\left\|u_{n}(s)-u_{m}(s)\right\|_{X^{1+\epsilon}}\right\} \\
& \quad+\left(2 \mu^{4} t^{\epsilon} \int_{0}^{t}(t-s)^{-1+4 \epsilon} s^{-5 \epsilon} d s\right) \sup _{0<s \leq t}\left\{s^{\epsilon}\left\|u_{n}(s)-u_{m}(s)\right\|_{X^{1+\epsilon}}\right\}
\end{aligned}
$$

which implies that for some $0<t \leq \tau_{0} \leq \tau_{1}$ we get

$$
t^{\epsilon}\left\|u_{n}(t)-u_{m}(t)\right\|_{X^{1+\epsilon}} \leq C\left\|u_{n}-u_{m}\right\|_{X^{1}} .
$$


A similar argument will show that

$$
\begin{aligned}
\left\|u_{n}(t)-u_{m}(t)\right\|_{X^{1}} & \leq C_{1} \sup _{0<s \leq t}\left\{s^{\epsilon}\left\|u_{n}(s)-u_{m}(s)\right\|_{X^{1+\epsilon}}\right\}+C_{2}\left\|u_{n}-u_{m}\right\|_{X^{1}} \\
& \leq C\left\|u_{n}-u_{m}\right\|_{X^{1}} .
\end{aligned}
$$

This will allow us to go to the limit as $n \rightarrow \infty$ and obtain solutions in the space $C\left(\left[0 \tau_{0}\right], X^{1}\right) \cap C\left(\left(0, \tau_{0}\right], X^{1+\epsilon}\right)$ with initial conditions in $X^{1}$.

From the discussion above, it seems reasonable to give the following definitions:

Definition 1. We say that $x:\left[t_{0}, \tau\right] \rightarrow X^{1}$ is an $\epsilon$-regular mild solution ( $\epsilon$-solution for short) to (1) if $x \in C\left(\left[t_{0}, \tau\right], X^{1}\right) \cap C\left(\left(t_{0}, \tau\right], X^{1+\epsilon}\right)$, and $x(t)$ satisfies

$$
x(t)=e^{A\left(t-t_{0}\right)} x_{0}+\int_{t_{0}}^{t} e^{A(t-s)} f(s, x(s)) d s .
$$

Definition 2. For $\epsilon \geq 0$, we will say that a map $g$ is an $\epsilon$-regular map relative to the pair $\left(X^{1}, X^{0}\right)$ if there exist $\rho>1, \gamma(\epsilon)$ with $\rho \epsilon \leq \gamma(\epsilon)<1$, and a constant $c$, such that $g: X^{1+\epsilon} \rightarrow X^{\gamma(\epsilon)}$ and

$$
\|g(x)-g(y)\|_{X^{\gamma(\epsilon)}} \leq c\|x-y\|_{X^{1+\epsilon}}\left(\|x\|_{X^{1+\epsilon}}^{\rho-1}+\|y\|_{X^{1+\epsilon}}^{\rho-1}+1\right) \quad \forall x, y \in X^{1+\epsilon} .
$$

The main results of this paper are contained in Section 2. They basically say that if $f(t, \cdot)$ is an $\epsilon$-regular map for some $\epsilon>0$, then we will have existence and uniqueness of $\epsilon$-regular mild solutions for problem (1) (see Theorem 1 or Corollary 1 for the autonomous case). This means that, in dealing with the problem of existence and uniqueness for a particular equation with critical nonlinearities, we need to do two things:

(i). Understand the scale of fractional power spaces associated to the linear operator $A$, especially the embeddings into known spaces like $L^{p}$ spaces.

(ii). Study the $\epsilon$-regularity properties of the nonlinearity $f$ in this scale of spaces. This is usually done using the Hölder inequality and Sobolev type embeddings.

Once (i) and (ii) are done, we can apply Theorem 1 and obtain existence and uniqueness results.

Moreover, it seems clear that the criticality of a particular nonlinearity $f$ is related to the $\epsilon$-regularity properties of $f$, and therefore we could classify the nonlinearities according to their $\epsilon$-regularity properties. This is done at the end of Section 2.

It is reasonable to think that the agenda explained above ((i),(ii) and Theorem 1) can be applied to many concrete problems - in particular, to the Navier-Stokes equations, the heat equation, systems of parabolic equations, strongly damped hyperbolic equations, etc. As examples we chose to study the Navier-Stokes equation in the Hilbert setting, and the heat equation in the $L^{q}$ and $W^{1, q}$ setting . This is done in Section 3. We recover several known results on existence and uniqueness of solutions for these equations, including those from the paper by Kato and Fujita $[\mathrm{KF}]$ for the Navier-Stokes equation and from the papers by Weissler [W1, W2] and Brezis and Cazenáve [BC]. All these very good papers were very inspiring for us, especially the last one.

The last section includes several comments about the uniqueness result obtained in Theorem 1 and its relation with other uniqueness and non-uniqueness results found in the literature $([\mathrm{BC}],[\mathrm{NS}],[\mathrm{LR}])$. Also, several open questions on the 
uniqueness problem are posed which we believe are very important for a full understanding of the subject.

Remark 1. After the paper was submitted for publication, it was pointed out to us by H. Amann that other scales of Banach spaces, different from the scale of fractional power spaces, could be used to deal with such problems. In connection with this, it is important to mention that for the abstract results presented in Section 2 the only requirements on the operator $A$ and the scale of spaces $\left\{X^{\alpha}\right\}_{0 \leq \alpha \leq 2}$ are that $-A$ is a sectorial operator and that (9) is satisfied. The proofs go through unchanged.

For the applications, and in order to solve critical problems, the scale must satisfy sharp embedding relations of the type (25). In the case of Dirichlet boundary conditions and $C^{2}$ domains these embedding relations are well known for the scale of fractional power spaces, thanks to [Tri, PS]. For other boundary conditions and more general operators the scale of fractional power spaces is not so well understood, so it may be better to use a different scale of Banach spaces for which these sharp embeddings are known. Some possibilities can be found in [AM2, AM3].

\section{ACKNOWLEDGMENTS}

We are indebted to Xu Yan Chen and T. Cazenave for several discussions related to this work. Also, we would like to thank S. M. Oliva, A. L. Pereira and L. A. Oliveira for all their comments and discussions about the results of this paper. Part of this work was done while the first author was visiting the ICMSC-USP at São Carlos, Brazil, and he would like to thank the members of the institute for their hospitality. Finally we are thankful to H. Amann for several comments on the scale of Banach spaces which lead to a considerable improvement of the results.

\section{Abstract Results}

With respect to the linear operator $A: D(A) \subset X^{0} \rightarrow X^{0}$ we will assume that $-A$ is a sectorial operator in the Banach space $X^{0}$. We will denote by $X^{\alpha}, \alpha \geq 0$, the fractional power spaces associated to the operator $A$ and by $e^{A t}$ the analytic semigroup generated by $A$. Without loss of generality we can assume that $e^{A t}$ is uniformly bounded. Let $M$ be such that

$$
t^{1+\alpha-\beta}\left\|e^{A t} x\right\|_{X^{1+\alpha}} \leq M\|x\|_{X^{\beta}}, \quad 0 \leq \beta \leq 1+\alpha \leq 2
$$

(see $[\mathrm{HE}])$.

With respect to the nonlinearities, let us consider the following class: with $\epsilon, \rho$, $\gamma(\epsilon)$ and $c$ positive constants, and $\nu(t)$ with $0 \leq \nu(t) \leq \delta, \lim _{t \rightarrow 0^{+}} \nu(t)=0$, define $\mathcal{F}:=\mathcal{F}(\epsilon, \rho, \gamma(\epsilon), c, \nu(\cdot))$ as the family of functions $f$ such that, for $t>0, f(t, \cdot)$ is an $\epsilon$-regular map relative to the pair $\left(X^{1}, X^{0}\right)$, satisfying

$$
\begin{gathered}
\|f(t, x)-f(t, y)\|_{X^{\gamma(\epsilon)}} \leq c\|x-y\|_{X^{1+\epsilon}}\left(\|x\|_{X^{1+\epsilon}}^{\rho-1}+\|y\|_{X^{1+\epsilon}}^{\rho-1}+\nu(t) t^{-\gamma(\epsilon)+\epsilon}\right), \\
\|f(t, x)\|_{X^{\gamma(\epsilon)}} \leq c\left(\|x\|_{X^{1+\epsilon}}^{\rho}+\nu(t) t^{-\gamma(\epsilon)}\right)
\end{gathered}
$$

for all $x, y \in X^{1+\epsilon}$.

Without loss of generality we can assume that the function $\nu(t)$ is non-decreasing.

In most cases in the argument below we will fix the parameters $\epsilon, \rho, \gamma(\epsilon)$ and $c$, and we will denote the class $\mathcal{F}$ defined above by $\mathcal{F}(\nu(\cdot))$. 
With these definitions we can now state the main result of this paper.

Theorem 1. Let $f \in \mathcal{F}(\epsilon, \rho, \gamma(\epsilon), c, \nu(\cdot))$. If $y_{0} \in X^{1}$, there exist $r>0$ and $\tau_{0}>0$ with the property that for any $x_{0} \in B_{X^{1}}\left(y_{0}, r\right)$ there exists a continuous function $x\left(\cdot, x_{0}\right):\left[0, \tau_{0}\right] \rightarrow X^{1}$, with $x(0)=x_{0}$, which is the unique $\epsilon$-regular mild solution starting at $x_{0}$ of the problem

$$
\begin{gathered}
\dot{x}=A x+f(t, x), \quad t>0, \\
x(0)=x_{0} .
\end{gathered}
$$

This solution satisfies

$$
\begin{gathered}
x \in C\left(\left(0, \tau_{0}\right], X^{1+\theta}\right), \quad 0 \leq \theta<\gamma(\epsilon), \\
t^{\theta}\left\|x\left(t, x_{0}\right)\right\|_{X^{1+\theta}} \stackrel{t \rightarrow 0^{+}}{\longrightarrow} 0, \quad 0<\theta<\gamma(\epsilon) .
\end{gathered}
$$

Moreover, if $x_{0}, z_{0} \in B_{X^{1}}\left(y_{0}, r\right)$, then

$t^{\theta}\left\|x\left(t, x_{0}\right)-x\left(t, z_{0}\right)\right\|_{X^{1+\theta}} \leq C\left\|x_{0}-z_{0}\right\|_{X^{1}}, \quad \forall t \in\left[0, \tau_{0}\right], 0 \leq \theta \leq \theta_{0}<\gamma(\epsilon)$.

Also, if $\gamma(\epsilon)>\rho \epsilon$, then $r$ can be chosen arbitrarily large. That is, the time of existence is uniform on bounded sets of $X^{1}$.

If $t \rightarrow f(t, x)$, as a map from $(0, \infty)$ to $X^{\gamma(\epsilon)}$, is locally Hölder continuous, uniformly on bounded sets of $x \in X^{1+\gamma(\epsilon)}$, then

$$
x \in C^{1}\left(\left(0, \tau_{0}\right], X^{\gamma(\epsilon)}\right) \cap C\left(\left(0, \tau_{0}\right], X^{1+\gamma(\epsilon)}\right),
$$

and $x\left(\cdot, x_{0}\right)$ is an strict solution of (12).

The constants above depend on the following: $\tau_{0}=\tau_{0}\left(y_{0}, A, \nu(\cdot), \epsilon, \rho, \gamma(\epsilon), c, M\right)$, $r=r\left(y_{0}, \epsilon, \rho, \gamma(\epsilon), c, M\right), C=C\left(\theta_{0}, \epsilon, \rho, \gamma(\epsilon), M\right)$.

In many applications the map $f$ is independent of time. For the shake of completeness and clarity we include in the following corollary the statement of Theorem 1 adapted to time-independent maps:

Corollary 1. Assume that $f$ is independent of time and that it is an $\epsilon$-regular map, for some $\epsilon>0$, relative to the pair $\left(X^{1}, X^{0}\right)$. Then, if $y_{0} \in X^{1}$, there exist $r=r\left(y_{0}\right)>0$ and $\tau_{0}=\tau_{0}\left(y_{0}\right)>0$ such that for $x_{0} \in X^{1}$ with $\left\|x_{0}-y_{0}\right\|_{X^{1}}<r$ there exists a continuous function $x:\left[0, \tau_{0}\right] \rightarrow X^{1}$, with $x(0)=x_{0}$, which is the unique $\epsilon$-regular mild solution to (3) starting at $x_{0}$. This solution satisfies

$$
\begin{gathered}
x \in C\left(\left(0, \tau_{0}\right], X^{1+\theta}\right), \quad 0 \leq \theta<\gamma(\epsilon), \\
t^{\theta}\left\|x\left(t, x_{0}\right)\right\|_{X^{1+\theta}} \stackrel{t \rightarrow 0^{+}}{\longrightarrow} 0, \quad 0<\theta<\gamma(\epsilon) .
\end{gathered}
$$

Moreover, if $x_{0}, z_{0} \in B_{X^{1}}\left(y_{0}, r\right)$, then

$t^{\theta}\left\|x\left(t, x_{0}\right)-x\left(t, z_{0}\right)\right\|_{X^{1+\theta}} \leq C\left(\theta_{0}\right)\left\|x_{0}-z_{0}\right\|_{X^{1}}, \quad \forall t \in\left[0, \tau_{0}\right], 0 \leq \theta \leq \theta_{0}<\gamma(\epsilon)$.

Also, if $\gamma(\epsilon)>\rho \epsilon$, then $r$ can be chosen arbitrarily large. That is, the time of existence can be chosen uniformly on bounded sets of $X^{1}$.

Furthermore, $x \in C^{1}\left(\left(0, \tau_{0}\right], X^{\gamma(\epsilon)}\right) \cap C\left(\left(0, \tau_{0}\right], X^{1+\gamma(\epsilon)}\right)$, that is, $x\left(\cdot, x_{0}\right)$ is an strict solution of (3).

The constants above depend on the following: $\tau_{0}=\tau_{0}\left(y_{0}, A, \epsilon, \rho, \gamma(\epsilon), c, M\right), r=$ $r\left(y_{0}, \epsilon, \rho, \gamma(\epsilon), c, M\right), C=C\left(\theta_{0}, \epsilon, \rho, \gamma(\epsilon), M\right)$.

The proof of this corollary is straightforward once we have proved Theorem 1 . 
Remark 2. Notice that we do not assume that $f$ is a well defined map on $X^{1}$. The only requirement on $f$ is that it is an $\epsilon$-regular map relative to $\left(X^{1}, X^{0}\right)$, for some $\epsilon>0$. In particular we can obtain an existence and uniqueness theorem in $X^{1}$ without the nonlinearity being defined on $X^{1}$.

Before we prove Theorem 1 we will need some lemmas.

Lemma 1. The operators $t^{\alpha} e^{-A t}: X^{1} \rightarrow X^{1+\alpha}, t>0$, are bounded linear operators satisfying $\left\|t^{\alpha} e^{-A t}\right\|_{L\left(X^{1}, X^{1+\alpha}\right)} \leq M$, with $M$ independent of $t$. Moreover, given a compact subset $J$ of $X^{1}$, we have

$$
\lim _{t \rightarrow 0^{+}} \sup _{x \in J}\left\|t^{\alpha} e^{-A t} x\right\|_{X^{1+\alpha}}=0 .
$$

Proof. The fact that $\left\|t^{\alpha} e^{-A t}\right\|_{L\left(X^{1}, X^{1+\alpha}\right)} \leq M$ comes from statement (9).

For the remaining part we just have to realize that the operators $t^{\alpha} e^{-A t}: X^{1} \rightarrow$ $X^{1+\alpha}$ are bounded, uniformly in $t$, that $\left\|t^{\alpha} e^{-A t} x\right\|_{X^{1+\alpha}} \stackrel{t \rightarrow 0^{+}}{\longrightarrow} 0$, for $x \in X^{1+\alpha}$, and that $X^{1+\alpha}$ is a dense subset of $X^{1}$.

Let us recall the definition of the beta function $\mathbf{B}(\cdot, \cdot):(0, \infty) \times(0, \infty) \rightarrow(0, \infty)$, which is

$$
\mathbf{B}(a, b)=\int_{0}^{1}(1-x)^{a-1} x^{b-1} d x .
$$

Define

$$
\mathbf{B}_{\epsilon}^{\theta}=\max _{0 \leq \xi \leq \theta}\{\mathbf{B}(\gamma(\epsilon)-\xi, 1-\gamma(\epsilon)), \mathbf{B}(\gamma(\epsilon)-\xi, 1-\rho \epsilon)\} .
$$

Lemma 2. Let $f \in \mathcal{F}(\nu(\cdot))$. If $x \in C\left((0, \tau], X^{1+\epsilon}\right)$, then, for all $0 \leq \theta<\gamma(\epsilon)$,

$$
t^{\theta}\left\|\int_{0}^{t} e^{A(t-s)} f(s, x(s)) d s\right\|_{X^{1+\theta}} \leq c M \boldsymbol{B}_{\epsilon}^{\theta}\left(\nu(t)+t^{\gamma(\epsilon)-\rho \epsilon}[\lambda(t)]^{\rho}\right), \quad 0<t \leq \tau,
$$

where $\lambda(t):=\sup _{s \in(0, t]}\left\{s^{\epsilon}\|x(s)\|_{X^{1+\epsilon}}\right\}$.

Proof. It is not difficult to see that

$$
\begin{aligned}
t^{\theta} \| & \int_{0}^{t} e^{A(t-s)} f(s, x(s)) d s\left\|_{X^{1+\theta}} \leq M t^{\theta} \int_{0}^{t}(t-s)^{-1+\gamma(\epsilon)-\theta}\right\| f(s, x(s)) \|_{X^{\gamma(\epsilon)}} d s \\
\leq & c M t^{\theta} \int_{0}^{t}(t-s)^{-1+\gamma(\epsilon)-\theta}\left(\nu(s) s^{-\gamma(\epsilon)}+\|x(s)\|_{X^{1+\epsilon}}^{\rho}\right) d s \\
\leq & c M t^{\theta} \nu(t) \int_{0}^{t}(t-s)^{-1+\gamma(\epsilon)-\theta} s^{-\gamma(\epsilon)} d s \\
& +c M t^{\theta} \int_{0}^{t}(t-s)^{-1+\gamma(\epsilon)-\theta} s^{-\rho \epsilon}\left[s^{\epsilon}\|x(s)\|_{X^{1+\epsilon}}\right]^{\rho} d s \\
\leq & c M \nu(t) \int_{0}^{1}(1-s)^{-1+\gamma(\epsilon)-\theta} s^{-\gamma(\epsilon)} d s \\
& +c M t^{\gamma(\epsilon)-\rho \epsilon}[\lambda(t)]^{\rho} \int_{0}^{1}(1-s)^{-1+\gamma(\epsilon)-\theta} s^{-\rho \epsilon} d s \\
= & c M \mathbf{B}_{\epsilon}^{\theta}\left[\nu(t)+t^{\gamma(\epsilon)-\rho \epsilon}[\lambda(t)]^{\rho}\right],
\end{aligned}
$$

from which the lemma follows. 
Lemma 3. Let $f \in \mathcal{F}(\nu(\cdot))$ and $x, y \in C\left((0, \tau], X^{1+\epsilon}\right)$ be such that $t^{\epsilon}\|x(t)\|_{X^{1+\epsilon}} \leq$ $\mu$ and $t^{\epsilon}\|y(t)\|_{X^{1+\epsilon}} \leq \mu$, for some $\mu>0$. Then, for all $0 \leq \theta<\gamma(\epsilon)$, we have

$$
t^{\theta}\left\|\int_{0}^{t} e^{A(t-s)}[f(s, x(s))-f(s, y(s))] d s\right\|_{X^{1+\theta}} \leq \Gamma_{\theta}(t) \sup _{s \in[0, \tau]} s^{\epsilon}\|x(s)-y(s)\|_{X^{1+\epsilon}}
$$

where

$$
\Gamma_{\theta}(t)=c M \boldsymbol{B}_{\epsilon}^{\theta}\left[\nu(t)+t^{\gamma(\epsilon)-\rho \epsilon} 2 \mu^{\rho-1}\right] .
$$

Proof. Using the $\epsilon$-regularity property of $f$, we have

$$
\begin{aligned}
& t^{\theta}\left\|\int_{0}^{t} e^{A(t-s)}[f(s, x(s))-f(s, y(s))] d s\right\|_{X^{1+\theta}} \\
& \leq t^{\theta} \int_{0}^{t} c M(t-s)^{-1+\gamma(\epsilon)-\theta}\|x(s)-y(s)\|_{X^{1+\epsilon}}\left(\nu(t) s^{-\gamma(\epsilon)+\epsilon}\right. \\
& \left.\quad+\|x(s)\|_{X^{1+\epsilon}}^{\rho-1}+\|y(s)\|_{X^{1+\epsilon}}^{\rho-1}\right) d s \\
& \leq c M t^{\theta} \nu(t) \int_{0}^{t}(t-s)^{-1+\gamma(\epsilon)-\theta} s^{-\gamma(\epsilon)} s^{\epsilon}\|x(s)-y(s)\|_{X^{1+\epsilon}} d s \\
& \quad+c M t^{\theta} \int_{0}^{t}(t-s)^{-1+\gamma(\epsilon)-\theta} s^{-\rho \epsilon}\left[\left(s^{\epsilon}\|x(s)\|_{X^{1+\epsilon}}\right)^{\rho-1}+\left(s^{\epsilon}\|y(s)\|_{X^{1+\epsilon}}\right)^{\rho-1}\right] \\
& \quad \times s^{\epsilon}\|x(s)-y(s)\|_{X^{1+\epsilon}} d s \\
& =\Gamma_{\theta}(t) \sup _{t \in[0, \tau]}\left\{s^{\epsilon}\|x(s)-y(s)\|_{\left.X^{1+\epsilon}\right\} .}\right.
\end{aligned}
$$

Proof of Theorem 1. We will divide the proof in two parts, existence and uniqueness.

Existence. Define $\mu$ by

$$
c M \mathbf{B}_{\epsilon}^{\epsilon} \mu^{\rho-1}=\frac{1}{8}
$$

and choose $r=r(\mu, M)>0$ such that

$$
r=\frac{\mu}{4 M}=\frac{1}{4 M\left(8 c M B_{\epsilon}^{\epsilon}\right)^{\frac{1}{\rho-1}}}
$$

Also, for $y_{0}$ fixed, choose $\tau_{0}=\tau_{0}\left(y_{0}, A, \mu, \nu(\cdot), \epsilon, \rho, \gamma(\epsilon), c, M\right) \in(0,1]$ such that $\nu(t)<\delta$ for $t \in\left(0, \tau_{0}\right]$ and

$$
\begin{gathered}
\left\|t^{\epsilon} e^{-A t} y_{0}\right\|_{X^{1+\epsilon}} \leq \frac{\mu}{2}, \quad 0 \leq t \leq \tau_{0}, \\
c M \delta \mathbf{B}_{\epsilon}^{\epsilon}=\min \left\{\frac{\mu}{8}, \frac{1}{4}\right\} .
\end{gathered}
$$

Notice that these choices imply that $\Gamma_{\epsilon}(t) \leq \frac{1}{2}$ for $t \in(0,1)$.

Since we will be looking for solutions which regularize immediately, we search for solutions in

$$
K\left(\tau_{0}\right)=\left\{x \in C\left(\left(0, \tau_{0}\right], X^{1+\epsilon}\right): \sup _{t \in\left(0, \tau_{0}\right]} t^{\epsilon}\|x(t)\|_{X^{1+\epsilon}} \leq \mu\right\} .
$$

with the norm

$$
\|x\|_{K\left(\tau_{0}\right)}=\sup _{t \in\left(0, \tau_{0}\right]} t^{\epsilon}\|x(t)\|_{X^{1+\epsilon}} .
$$


Assume that $x_{0} \in X^{1}$ with $\left\|x_{0}-y_{0}\right\|_{X^{1}}<r$ and on $K\left(\tau_{0}\right)$ define the map

$$
(T x)(t)=e^{A t} x_{0}+\int_{0}^{t} e^{A(t-s)} f(s, x(s)) d s .
$$

We will show that, for all $x_{0} \in B_{X^{1}}\left(y_{0}, r\right), T$ takes $K\left(\tau_{0}\right)$ into itself, and that $T$ is a strict contraction in $K\left(\tau_{0}\right)$.

Let us first prove that $T$ is a well-defined map and that $T\left(K\left(\tau_{0}\right)\right) \subset K\left(\tau_{0}\right)$. We start by showing that

$$
\text { if } x \in K\left(\tau_{0}\right) \text {, then } T x \in C\left(\left(0, \tau_{0}\right], X^{1+\theta}\right), \quad \forall \theta \in[0, \gamma(\epsilon)) .
$$

Fix $t_{2} \in\left(0, \tau_{0}\right]$ and let $\tau_{0} \geq t_{1}>t_{2}$; then, for $0 \leq \theta<\gamma(\epsilon)$, we have

$$
\begin{aligned}
&\left\|(T x)\left(t_{1}\right)-(T x)\left(t_{2}\right)\right\|_{X^{1+\theta}} \\
& \leq\left\|\left(e^{-A t_{1}}-e^{-A t_{2}}\right) x_{0}\right\|_{X^{1+\theta}}+\left\|\int_{t_{2}}^{t_{1}} e^{A\left(t_{1}-s\right)} f(s, x(s)) d s\right\|_{X^{1+\theta}} \\
&+\left\|\left[I-e^{-A\left(t_{1}-t_{2}\right)}\right] \int_{0}^{t_{2}} e^{A\left(t_{2}-s\right)} f(s, x(s)) d s\right\|_{X^{1+\theta}} .
\end{aligned}
$$

In the above, the first and third term trivially go to zero as $t_{1} \rightarrow t_{2}$. Let us consider the second term. For it we have

$$
\begin{aligned}
& \left\|\int_{t_{2}}^{t_{1}} e^{A\left(t_{1}-s\right)} f(s, x(s)) d s\right\|_{X^{1+\theta}} \\
& \leq c \int_{t_{2}}^{t_{1}} M\left(t_{1}-s\right)^{-1+\gamma(\epsilon)-\theta}\left(\delta s^{-\gamma(\epsilon)}+\|x(s)\|_{X^{1+\epsilon}}^{\rho}\right) d s \\
& \leq c M \delta \int_{t_{2}}^{t_{1}}\left(t_{1}-s\right)^{-1+\gamma(\epsilon)-\theta} s^{-\gamma(\epsilon)} d s \\
& \quad+c M \int_{t_{2}}^{t_{1}}\left(t_{1}-s\right)^{-1+\gamma(\epsilon)-\theta} s^{-\rho \epsilon}\left(s^{\epsilon}\|x(s)\|_{X^{1+\epsilon}}\right)^{\rho} d s \\
& \quad \leq M \delta t_{1}^{-\theta} \int_{t_{2} / t_{1}}^{1}(1-s)^{-1+\gamma(\epsilon)-\theta} s^{-\gamma(\epsilon)} d s \\
& \quad+c M \mu^{\rho} t_{1}^{\gamma(\epsilon)-\theta-\rho \epsilon} \int_{t_{2} / t_{1}}^{1}(1-s)^{-1+\gamma(\epsilon)-\theta} s^{-\rho \epsilon} d s,
\end{aligned}
$$

which goes to zero as $t_{1} \rightarrow t_{2}^{+}$. The case $t_{1}<t_{2}$ is similar.

Let us now show that $t^{\epsilon}\|x(t)\|_{X^{1+\epsilon}} \leq \mu$, for all $t \in\left(0, \tau_{0}\right]$. In fact,

$$
\begin{aligned}
& t^{\epsilon}\|(T x)(t)\|_{X^{1+\epsilon}} \\
& \leq\left\|t^{\epsilon} e^{-A t} x_{0}\right\|_{X^{1+\epsilon}}+c M t^{\epsilon} \int_{0}^{t}(t-s)^{-1+\gamma(\epsilon)-\epsilon}\left(\delta s^{-\gamma(\epsilon)}+\|x\|_{X^{1+\epsilon}}^{\rho}\right) d s \\
& \leq\left\|t^{\epsilon} e^{-A t} x_{0}\right\|_{X^{1+\epsilon}}+c M t^{\epsilon} \delta \int_{0}^{t}(t-s)^{-1+\gamma(\epsilon)-\epsilon} s^{-\gamma(\epsilon)} d s \\
&+c M t^{\epsilon} \int_{0}^{t}(t-s)^{-1+\gamma(\epsilon)-\epsilon} s^{-\rho \epsilon}\left(s^{\epsilon}\|x\|_{X^{1+\epsilon}}\right)^{\rho} d s \\
& \leq\left\|t^{\epsilon} e^{-A t} x_{0}\right\|_{X^{1+\epsilon}}+c M \delta \mathbf{B}_{\epsilon}^{\epsilon}+c M \mathbf{B}_{\epsilon}^{\epsilon} \mu^{\rho} \\
& \leq M r+\left\|t^{\epsilon} e^{-A t} y_{0}\right\|_{X^{1+\epsilon}}+c M \mathbf{B}_{\epsilon}^{\epsilon} \delta+c M \mathbf{B}_{\epsilon}^{\epsilon} \mu^{\rho} \leq \mu .
\end{aligned}
$$

This shows that $T$ takes $K\left(\tau_{0}\right)$ into itself. 
The next step is to prove that the map $T$ is a contraction from $K\left(\tau_{0}\right)$ into itself.

It follows from Lemma 3, by taking $\theta=\epsilon$, that $T$ is a strict contraction in $K\left(\tau_{0}\right)$ and that

$$
\|T(x)-T(y)\|_{K\left(\tau_{0}\right)} \leq \frac{1}{2}\|x-y\|_{K\left(\tau_{0}\right)} .
$$

By the Banach contraction principle we have that $T$ has a unique fixed point in $K\left(\tau_{0}\right)$. We will denote this fixed point by $X\left(t, x_{0}\right)$; it is defined for $\left\|x_{0}-y_{0}\right\|_{X^{1}}<r$, $0 \leq t \leq \tau_{0}$. Note that, from (15), $X\left(\cdot, x_{0}\right) \in C\left(\left(0, \tau_{0}\right], X^{1+\theta}\right)$ for all $0 \leq \theta<\gamma(\epsilon)$.

Let us prove that $t^{\theta}\left\|X\left(t, x_{0}\right)\right\|_{X^{1+\theta}} \rightarrow 0$ as $t \rightarrow 0$ for all $0<\theta<\gamma(\epsilon)$.

From Lemma 2,

$$
\begin{gathered}
t^{\theta}\left\|X\left(t, x_{0}\right)\right\|_{X^{1+\theta}} \leq t^{\theta}\left\|e^{A t} x_{0}\right\|_{X^{1+\theta}}+t^{\theta} \int_{0}^{t}\left\|e^{A(t-s)} f\left(s, X\left(s, x_{0}\right)\right)\right\|_{X^{1+\theta}} d s \\
\leq t^{\theta}\left\|e^{A t} x_{0}\right\|_{X^{1+\theta}}+c M \mathbf{B}_{\epsilon}^{\theta} \nu(t)+c M \mathbf{B}_{\epsilon}^{\theta} \mu^{\rho-1} \sup _{0<s \leq t}\left\{t^{\epsilon}\left\|X\left(t, x_{0}\right)\right\|_{X^{1+\epsilon}}\right\} .
\end{gathered}
$$

Therefore if $\theta=\epsilon$ we have

$$
t^{\epsilon}\left\|X\left(t, x_{0}\right)\right\|_{X^{1+\epsilon}} \leq t^{\epsilon}\left\|e^{A t} x_{0}\right\|_{X^{1+\epsilon}}+c M \mathbf{B}_{\epsilon}^{\epsilon} \nu(t)+\frac{1}{8} \sup _{0<s \leq t}\left\{t^{\epsilon}\left\|X\left(t, x_{0}\right)\right\|_{X^{1+\epsilon}}\right\},
$$

from which we obtain

$$
\sup _{0<s \leq t}\left\{s^{\epsilon}\left\|X\left(s, x_{0}\right)\right\|_{X^{1+\epsilon}}\right\} \leq \frac{8}{7}\left(t^{\epsilon}\left\|e^{A t} x_{0}\right\|_{X^{1+\epsilon}}+c M \mathbf{B}_{\epsilon}^{\epsilon} \nu(t)\right) \rightarrow 0 \quad \text { as } t \rightarrow 0 .
$$

If $0<\theta<\gamma(\epsilon)$, from the above expressions we also obtain $t^{\theta}\left\|X\left(t, x_{0}\right)\right\|_{X^{1+\theta}} \rightarrow 0$ as $t \rightarrow 0$.

Let us prove now that

$$
\lim _{t \rightarrow 0^{+}}\left\|X\left(t, x_{0}\right)-x_{0}\right\|_{X^{1}}=0 .
$$

In fact, from Lemma 2

$$
\begin{aligned}
& \left\|X\left(t, x_{0}\right)-x_{0}\right\|_{X^{1}} \leq\left\|e^{A t} x_{0}-x_{0}\right\|_{X^{1}}+\int_{0}^{t}\left\|e^{A(t-s)} f\left(s, X\left(s, x_{0}\right)\right)\right\|_{X^{1}} d s \\
& \leq\left\|e^{A t} x_{0}-x_{0}\right\|_{X^{1}}+c M \mathbf{B}_{\epsilon}\left(\nu(t)+\left[\sup _{0<s \leq t}\left\{t^{\epsilon}\left\|X\left(t, x_{0}\right)\right\|_{X^{1+\epsilon}}\right\}\right]^{\rho}\right) \stackrel{t \rightarrow 0^{+}}{\longrightarrow} 0 .
\end{aligned}
$$

From all this we see that $X\left(t, x_{0}\right)$ is an $\epsilon$-regular solution starting at $x_{0}$ and it is the unique $\epsilon$-regular solution starting at $x_{0}$, in the set $K\left(\tau_{0}\right)$. We will hereafter call it the $K$-solution starting at $x_{0}$.

Moreover, if $x_{0}, z_{0} \in B_{X^{1}}\left(y_{0}, r\right)$, taking into account the estimates of Lemma 3 and our choice of $\tau_{0}$, we have

$$
\begin{aligned}
t^{\theta} \| X( & \left.t, x_{0}\right)-X\left(t, z_{0}\right) \|_{X^{1+\theta}} \\
\leq & t^{\theta}\left\|e^{A t}\left(x_{0}-z_{0}\right)\right\|_{X^{1+\theta}} \\
& +t^{\theta} \int_{0}^{t}\left\|e^{A(t-s)}\left[f\left(s, X\left(s, x_{0}\right)\right)-f\left(s, X\left(s, z_{0}\right)\right)\right]\right\|_{X^{1+\theta}} d s \\
\leq & M\left\|x_{0}-z_{0}\right\|_{X^{1}}+\Gamma_{\theta}(t) \sup _{s \in\left[0, \bar{\tau}_{0}\right]} s^{\epsilon}\left\|X\left(s, x_{0}\right)-X\left(s, z_{0}\right)\right\|_{X^{1+\epsilon}} .
\end{aligned}
$$


For $\theta=\epsilon$ we get

$$
\begin{aligned}
& t^{\epsilon}\left\|X\left(t, x_{0}\right)-X\left(t, z_{0}\right)\right\|_{X^{1+\epsilon}} \\
& \quad \leq M\left\|x_{0}-z_{0}\right\|_{X^{1}}+\frac{1}{2} \sup _{s \in\left[0, \bar{\tau}_{0}\right]} s^{\epsilon}\left\|X\left(s, x_{0}\right)-X\left(s, z_{0}\right)\right\|_{X^{1+\epsilon}}
\end{aligned}
$$

which implies

$$
t^{\epsilon}\left\|X\left(t, x_{0}\right)-X\left(t, z_{0}\right)\right\|_{X^{1+\epsilon}} \leq 2 M\left\|x_{0}-z_{0}\right\|_{X^{1}} .
$$

For $0 \leq \theta \leq \theta_{0}<\gamma(\epsilon)$ we have from (16) that

$$
\begin{aligned}
t^{\theta}\left\|X\left(t, x_{0}\right)-X\left(t, z_{0}\right)\right\|_{X^{1+\theta}} & \leq M\left\|x_{0}-z_{0}\right\|_{X^{1}}+\Gamma_{\theta}(t) 2 M\left\|x_{0}-z_{0}\right\|_{X^{1}} \\
& \leq C\left(\theta_{0}\right)\left\|x_{0}-z_{0}\right\|_{X^{1}},
\end{aligned}
$$

where $C\left(\theta_{0}\right)=M\left(1+2 \sup \left\{\Gamma_{\theta}(t) ; t \in\left[0, \tau_{0}\right], 0 \leq \theta \leq \theta_{0}\right\}\right)$.

This concludes the existence part of the theorem.

Uniqueness. Notice that from the existence part we have that for any $x_{0} \in B_{X^{1}}\left(y_{0}, r\right)$ and for any $f \in \mathcal{F}(\nu(\cdot))$ there exists a unique $K$-solution, defined in $\left[0, \tau_{0}\right]$, of the problem

$$
\begin{gathered}
\dot{x}=A x+f(t, x), \\
x(0)=x_{0} .
\end{gathered}
$$

To stress the dependence of the $K$-solution on $f$ we will denote it by $X_{f}\left(t, x_{0}\right)$.

Consider the following:

Lemma 4. If $\phi(t)$ is an $\epsilon$-regular solution in $\left[0, t_{0}\right]$ of (17) and $t^{\epsilon}\|\phi(t)\|_{X^{1+\epsilon}} \rightarrow 0$ as $t \rightarrow 0$, then $\phi(t)=X_{f}\left(t, x_{0}\right)$ for all $0 \leq t \leq \min \left\{\tau_{0}, t_{0}\right\}$

Proof. It is clear that $\phi \in K(\bar{\tau})$ for some small $\bar{\tau} \leq \tau_{0}$. Since we also have $X_{f}\left(\cdot, x_{0}\right) \in K(\bar{\tau})$ and both $\phi$ and $X_{f}\left(\cdot, x_{0}\right)$ are solutions of the integral equation, we get $X_{f}\left(t, x_{0}\right)=\phi(t)$ for all $0 \leq t \leq \bar{\tau}$. With a standard continuation argument it is easy to see that we must have $X_{f}\left(t, x_{0}\right)=\phi(t)$ for all $0 \leq t \leq \min \left\{t_{0}, \tau_{0}\right\}$. This proves the lemma.

Lemma 5. If $f \in \mathcal{F}(\nu(\cdot))$, then $f_{a} \in \mathcal{F}\left(\nu_{a}(\cdot)\right)$ for all $a \geq 0$, where $f_{a}(t, x) \equiv$ $f(t+a, x)$, and $\nu_{a}(t)=\nu(t+a)(t / t+a)^{\gamma(\epsilon)} \leq \nu(t+a)$. Moreover, there exists an $a_{0}>0$ so small that for all $a \in\left[0, a_{0}\right]$ the time of existence $\tau_{0}(a)$ given by (14) can be chosen independent of $a$.

Proof. The first part of the lemma is trivial.

For the second one we just need to observe that if $\nu(t)<\delta$ for $t \in\left[0, \tau_{0}\right]$, then, for small $a$, we will also have $\nu(t+a)<\delta$ for $t \in\left[0, \tau_{0}\right]$.

Following similar ideas as in [BC], we can now prove the uniqueness of $\epsilon-$ regular solutions.

Let $\phi(t), 0 \leq t \leq t_{0}$, be an $\epsilon$-regular solution starting in $x_{0} \in B_{X^{1}}\left(y_{0}, r\right)$. Since $\phi \in C\left(\left[0, t_{0}\right], X^{1}\right)$, there exists $a_{0} \in\left(0, t_{0}\right]$ such that $\phi(a) \in B_{X^{1}}\left(y_{0}, r\right)$, for all $0<a \leq a_{0}$. Notice that $\phi_{a}(\cdot) \equiv \phi(a+\cdot) \in C\left(\left[0, t_{0}-a\right], X^{1+\epsilon}\right)$, and therefore $t^{\epsilon}\left\|\phi_{a}(t)\right\|_{X^{1+\epsilon}} \rightarrow 0$ as $t \rightarrow 0$. Moreover, $\phi_{a}$ is an $\epsilon$-regular mild solution of

$$
\begin{gathered}
\dot{x}=A x+f_{a}(t, x), \\
x(0)=\phi(a) .
\end{gathered}
$$


From Lemma 5 and the results of the existence part we have that there exists a unique $K$-solution of problem (18), $X_{f_{a}}(t, \phi(a))$, defined in $\left[0, \tau_{0}\right]$. Moreover, from Lemma 4, we get $X_{f_{a}}(t, \phi(a))=\phi_{a}(t)$ for all $0 \leq t \leq \min \left\{\tau_{0}, t_{0}-a\right\}$, for all $0<a \leq a_{0}$. In particular this implies that without loss of generality we can assume that $t_{0} \geq \tau_{0}$, since if this is not the case we can define $\tilde{\phi}(t)=\phi(t)$ for $0 \leq t \leq t_{0}$ and $\tilde{\phi}(t)=X_{f_{a_{0}}}\left(t-a_{0}, \phi\left(a_{0}\right)\right)$ for $t_{0} \leq t \leq \tau_{0}$, and from the results above $\tilde{\phi}$ is also an $\epsilon$-regular solution starting at $x_{0}$.

In view of the definition of a $K$-solution, the only thing we need to show is that $t^{\epsilon}\|\phi(t)\|_{X^{1+\epsilon}} \leq \mu$ for all $0<t \leq \tau_{0}$. But, for $0<a<a_{0}$,

$$
\begin{aligned}
t^{\epsilon}\|\phi(t)\|_{X^{1+\epsilon}} & \leq t^{\epsilon}\|\phi(t)-\phi(t+a)\|_{X^{1+\epsilon}}+t^{\epsilon}\left\|X_{f_{a}}(t, \phi(a))\right\|_{X^{1+\epsilon}} \\
& \leq t^{\epsilon}\|\phi(t)-\phi(t+a)\|_{X^{1+\epsilon}}+\mu .
\end{aligned}
$$

For $0<t \leq \tau_{0}$ fixed, letting $a \rightarrow 0$ we have that $t^{\epsilon}\|\phi(t)-\phi(t+a)\|_{X^{1+\epsilon}} \rightarrow 0$, which implies that $t^{\epsilon}\|\phi(t)\|_{X^{1+\epsilon}} \leq \mu$ for all $t \in\left(0, \tau_{0}\right]$. This concludes the uniqueness part of the theorem.

For the case where $\gamma(\epsilon)>\rho \epsilon$, we proceed as follows. Let us define $y(t)=x(a t)$, for some $a<1$. The equation for $y$ is $\dot{y}=\tilde{A} y+\tilde{f}(t, y)$, where $\tilde{f}(t, x)=a f(a t, x)$, $\tilde{A}=a A$. Moreover, notice that $x(t)$ is a solution of the original equation in $\left(0, \tau_{0}\right]$ if and only if $y(t), t \in\left[0, a \tau_{0}\right]$, is a solution of the new equation. For this new equation, applying the existence part of the theorem, we can find a positive number $\tilde{r}$ such that the conclusions of the theorem are valid. Notice that from (13) we have

$$
\tilde{r}=\frac{1}{4 \tilde{M}\left(8 \tilde{c} \tilde{M} B_{\epsilon}^{\epsilon}\right)^{\frac{1}{\rho-1}}}
$$

where $\tilde{r}, \tilde{c}$ and $\tilde{M}$ are constants related to the new equations. Let us relate $\tilde{r}, \tilde{c}, \tilde{M}$ with $r, c$ and $M$. Denote by $\tilde{X}^{\alpha}$ the fractional power spaces associated to the operator $\tilde{A}$. Note that $\|\cdot\|_{\tilde{X}^{\alpha}}=a^{\alpha}\|\cdot\|_{X^{\alpha}}$ and

$$
t^{\alpha-\beta}\left\|e^{a A t} x\right\|_{\tilde{X}^{\alpha}}=a^{\beta}(a t)^{\alpha-\beta}\left\|e^{A a t} x\right\|_{X^{\alpha}} \leq M a^{\beta}\|x\|_{X^{\beta}}=M\|x\|_{\tilde{X}^{\beta}},
$$

which implies that $\tilde{M}=M$. To see that $\tilde{c}=a^{\gamma(\epsilon)-\rho \epsilon+1-\rho} c$, observe that

$$
\begin{aligned}
\|\tilde{f}(t, y)\|_{\tilde{X}^{\gamma(\epsilon)}} & =a^{\gamma(\epsilon)+1}\|f(a t, y)\|_{X^{\gamma(\epsilon)}} \\
& \leq a^{\gamma(\epsilon)+1-\rho(1+\epsilon)} c\left(\nu(a t) a^{\rho(1+\epsilon)}(a t)^{-\gamma(\epsilon)}+\|y\|_{\tilde{X}^{1+\epsilon}}^{\rho}\right) \\
& \leq a^{\gamma(\epsilon)+1-\rho(1+\epsilon)} c\left(\tilde{\nu}(t) t^{-\gamma(\epsilon)}+\|y\|_{\tilde{X}^{1+\epsilon}}^{\rho}\right),
\end{aligned}
$$

where $\tilde{\nu}(t)=\nu(a t) a^{\rho(1+\epsilon)-\gamma(\epsilon)}$. The computations with the Lipschitz properties of $f$ are similar. From this we have

$$
\tilde{r}=\frac{1}{4 \tilde{M}\left(8 \tilde{c} \tilde{M} B_{\epsilon}^{\epsilon}\right)^{\frac{1}{\rho-1}}}=r a^{(\rho \epsilon-\gamma(\epsilon)) /(\rho-1)+1} .
$$

This implies that if $\left\|y_{0}-x_{0}\right\|_{\tilde{X}^{1}}<\tilde{r}$ there exists a $\tilde{\tau}_{0}$ such that the solution of $\dot{\tilde{x}}=\tilde{A} \tilde{x}+\tilde{f}(t, \tilde{x})$ starting in $x_{0}$ is defined in $\left[0, \tilde{\tau}_{0}\right]$. Therefore, if

$$
\left\|y_{0}-x_{0}\right\|_{X^{1}}<r a^{(\rho \epsilon-\gamma(\epsilon)) /(\rho-1)},
$$


then $\dot{x}=A x+f(t, x)$ has the solution $x\left(t, x_{0}\right)=\tilde{x}\left(\frac{t}{a}, x_{0}\right)$ defined in $\left[0, a \tilde{\tau}_{0}\right]$. Since $a$ can be chosen arbitrarily small and $\gamma(\epsilon)>\rho \epsilon$, we see that solutions have a common interval of existence on bounded subsets of $X^{1}$.

Finally, if $t \rightarrow f(t, x)$ is locally Hölder continuous for $t>0$, uniformly on bounded sets of $X^{1+\epsilon}$, by standard regularity arguments (see for example [HE]) we obtain the regularity stated in the theorem.

This concludes the proof of the theorem.

From Theorem 1 we have the following

Corollary 2. If $f$ is as in Theorem 1 and if $K$ is a precompact set in $X^{1}$, then there exists a $\tau_{0}=\tau(K)$ such that the $\epsilon$-regular solution starting at $x_{0}$ exists for time $\tau_{0}$ for any $x_{0} \in K$

Proof. By Theorem 1, for any $y_{0} \in \bar{K} \equiv C l(K)$ there exist a $r\left(y_{0}\right)$ and a $\tau\left(y_{0}\right)$ such that for any $x_{0} \in X^{1}$ with $\left\|x_{0}-y_{0}\right\|_{X^{1}}<r\left(y_{0}\right)$ the unique $\epsilon$-regular solution exists in $\left[0, \tau\left(y_{0}\right)\right]$. By the compactness of $\bar{K}$ we can choose $y_{1}, \cdots, y_{n} \in \bar{K}$ such that $K \subset \bigcup B_{X^{1}}\left(y_{i}, r\left(y_{i}\right)\right)$. Choosing $\tau_{0}=\min \left\{\tau\left(y_{i}\right): 1 \leq i \leq n\right\}$, we prove the corollary.

We now prove a result on the maximal time of existence of $\epsilon$-regular solutions.

Proposition 1. If $f$ is as in Theorem 1 and $x\left(t, x_{0}\right)$ is an $\epsilon$-regular solution starting at $x_{0}$ with a maximal time of existence $\tau_{m}<\infty$, then $\lim _{t \rightarrow \tau_{m}^{-}}\left\|x\left(t, x_{0}\right)\right\|_{X^{1+\delta}}=$ $\infty$, for any $0<\delta<\epsilon$. If, moreover, $\gamma(\epsilon)>\rho \epsilon$, then also $\lim _{t \rightarrow \tau_{m}^{-}}\left\|x\left(t, x_{0}\right)\right\|_{X^{1}}=\infty$.

Proof. It is easy to check that if $f$ is $\epsilon$-regular relative to $\left(X^{1}, X^{0}\right)$ with $\gamma(\epsilon) \geq \rho \epsilon$, then for any $0<\delta<\epsilon, f$ is $\epsilon^{*}$-regular relative to $\left(X^{1+\delta}, X^{\delta}\right)$, for $\epsilon^{*}=\epsilon-\delta$, with $\gamma\left(\epsilon^{*}\right)>\rho \epsilon^{*}$. Also, if $\gamma(\epsilon)>\rho \epsilon$, then we can take $\delta=0$. Assume the solution remains bounded in $X^{1+\delta}$ along a sequence that converges to $\tau_{m}$. Then, using Theorem 1 for the pair $\left(X^{1+\delta}, X^{\delta}\right)$ and the uniform existence time on bounded sets, we get that the solution can be extended beyond $\tau_{m}$, which is a contradiction. This proves the proposition.

In the autonomous case, $f$ is often an $\epsilon$-regular map for a range of values of the parameter $\epsilon$. In this direction we have the following:

Corollary 3. If $f$ is an $\epsilon$-regular map for all $\epsilon \in\left(\epsilon_{0}, \epsilon_{1}\right]$ and if we denote by $x_{\epsilon}\left(t, x_{0}\right)$ the unique $\epsilon$-regular solution starting at $x_{0}$, for $\epsilon \in\left(\epsilon_{0}, \epsilon_{1}\right]$, then $x_{\epsilon}=x_{\epsilon_{1}}$ and $x \in C\left((0, \tau], X^{1+\gamma\left(\epsilon_{1}\right)}\right)$.

If $f$ is a time-independent map which is $\epsilon$-regular, for $\epsilon \in I$, relative to the pair $\left(X^{1}, X^{0}\right)$, we classify the map in the following way:

- If $I=\left[0, \epsilon_{1}\right]$ for some $\epsilon_{1}>0$ and $\gamma(0)>0$, we say that $f$ is a subcritical map relative to $\left(X^{1}, X^{0}\right)$.

- If $I=\left[0, \epsilon_{1}\right]$ for some $\epsilon_{1}>0$ with $\gamma(\epsilon)=\rho \epsilon, \epsilon \in I$, and if $f$ is not subcritical, then we say that $f$ is a critical map relative to $\left(X^{1}, X^{0}\right)$.

- If $I=\left(0, \epsilon_{1}\right]$ for some $\epsilon_{1}>0$ with $\gamma(\epsilon)=\rho \epsilon, \epsilon \in I$, and $f$ is not subcritical or critical, then we say that $f$ is a double-critical map relative to $\left(X^{1}, X^{0}\right)$.

- If $I=\left[\epsilon_{0}, \epsilon_{1}\right]$ for some $\epsilon_{1}>\epsilon_{0}>0$ with $\gamma\left(\epsilon_{0}\right)>\rho \epsilon_{0}$ and $f$ is not subcritical, critical or double critical, then we say that $f$ is an ultra-subcritical map relative to $\left(X^{1}, X^{0}\right)$. 
- If $I=\left[\epsilon_{0}, \epsilon_{1}\right]$ for some $\epsilon_{1}>\epsilon_{0}>0$ with $\gamma(\epsilon)=\rho \epsilon, \epsilon \in I$, and if $f$ is not subcritical, critical, double critical or ultra-subcritical, then we say that $f$ is an ultra-critical map relative to $\left(X^{1}, X^{0}\right)$.

Note that if $f$ is subcritical then $f: X^{1} \rightarrow X^{\gamma(0)}, \gamma(0)>0$, which is the usual definition of subcritical map. When $f$ is a critical map it takes $X^{1}$ into $X^{0}$ but there is no positive constant $\alpha$ such that $f$ takes $X^{1}$ into $X^{\alpha}$. When $f$ is double-critical (this name first appears in $[\mathrm{BC}]$ ) it is not defined as a map from $X^{1}$ into $X^{0}$ but it is $\epsilon$-regular for arbitrarily small positive values of $\epsilon$. When $f$ is ultra-subcritical or ultra-critical it is not a well defined map in $X^{1+\epsilon}$ for small values of $\epsilon>0$, and it is only an $\epsilon$-regular map when $\epsilon \geq \epsilon_{0}>0$, for some $\epsilon_{0}$. The main difference between ultra-subcritical and ultra-critical maps is that for the former the time of existence of the solution can be chosen uniformly on bounded sets of $X^{1}$, while for the latter this is still an unknown property.

In Section 3 we will supply several examples of nonlinearities, coming from the Navier-Stokes and heat equations, and will classify them according to the above scheme.

\section{Applichtions}

It is clear from the results in the previous section that for a given problem $\dot{x}=A x+f(t, x)$, where $A$ is a sectorial operator with fractional powers $X^{\alpha}, \alpha \in \mathbb{R}$, we need to study these fractional power spaces and the $\epsilon$-regularity properties of the map $f$. In this way the local existence of solutions for this problem is reduced to a good knowledge of the linear operator $A$.

The characterization of fractional power spaces is a very interesting and difficult subject. For the cases considered here (that is, the Stokes operator in the Hilbert setting and the Dirichlet Laplacian in $\left.L^{p}(\Omega), 1<p<\infty\right)$, these characterizations are well known (see [vW] for the Stokes operator and [Tri, p. 103], [PS] for the Dirichlet Laplacian in a $C^{2}$ domain). These characterizations are not so well known for other elliptic operators and boundary conditions, but there are results with more regular coefficients and domains (see, for example $[\mathrm{S}, \mathrm{Fu}]$ ). We point out that for the results presented here we do not need a complete characterization of the fractional power spaces, but rather its embedding relations with the $L^{p}(\Omega)$ spaces (see (22), $(25))$.

In the following examples we show how this technique considerably simplifies the study of local existence in parabolic equations and Navier-Stokes equations with critically growing nonlinearities as seen in [KF, FK, BC, W1, W2].

In this section we will constantly use certain well known embeddings that we summarize as:

$$
\begin{aligned}
& H_{p_{1}}^{l_{1}}(\Omega) \hookrightarrow H_{p_{2}}^{l_{2}}(\Omega), \quad \text { if } \frac{l_{1}}{N}-\frac{1}{p_{1}} \geq \frac{l_{2}}{N}-\frac{1}{p_{2}}, 1<p_{1} \leq p_{2}<\infty, \\
& H_{p}^{l}(\Omega) \hookrightarrow C^{\eta}(\bar{\Omega}), \quad \text { if } l-\frac{N}{p}>\eta>0,
\end{aligned}
$$

where the spaces $H_{p}^{l}(\Omega)$ are the Bessel potentials, also called Lebesgue spaces (see [AD]). Notice that $H_{p}^{l}(\Omega)=W^{l, p}(\Omega)$, the standard Sobolev-Slobodeckii spaces, whenever $p=2$ and $l \in \mathbb{R}$, or $p>1$ and $l$ is an integer (see $[\mathrm{AD}]$ ). 
3.1. Navier-Stokes Equations. Consider the $N$-dimensional Navier-Stokes system; that is, if $\Omega \subset \mathbb{R}^{N}$ is a bounded smooth domain,

$$
\begin{aligned}
& u_{t}=\Delta u-\nabla p+g-(u \cdot \nabla) u, \quad x \in \Omega, \\
& \operatorname{div}(u)=0, \quad x \in \Omega, \\
& u=0, \quad x \in \partial \Omega
\end{aligned}
$$

where $u \in \mathbb{R}^{N}$ is the velocity field, $p$ is the pressure and $g$ is the external force.

It is a standard procedure to set this problem in an abstract context by using the orthogonal projection $P: L^{2}\left(\Omega, \mathbb{R}^{N}\right) \rightarrow H$, where $H$ is the closure of $\{u \in$ $\left.C^{2}\left(\Omega, \mathbb{R}^{N}\right): \operatorname{div}(u)=0, u \cdot n=0\right\}$ in $L^{2}\left(\Omega, \mathbb{R}^{N}\right)$. In this way the problem becomes

$$
\dot{u}=A u+f(u)+h(t),
$$

where $A: D(A) \subset H \rightarrow H, D(A)=H^{2}\left(\Omega, \mathbb{R}^{N}\right) \cap V, V=\left\{u \in H_{0}^{1}\left(\Omega, \mathbb{R}^{N}\right)\right.$ : $\operatorname{div}(u)=0\}, A=P \Delta, f(u)=-P(u \cdot \nabla) u$ and $h=P g$. The operator $A$ is self-adjoint and positive.

In their very nice papers $[\mathrm{KF}, \mathrm{FK}]$, Fujita and Kato search for the largest fractional power space in which a local-existence and uniqueness theorem for the problem (21) can be proved, for $N=3$. They arrive at the following result: If $u_{0} \in D\left(A^{\frac{1}{4}}\right)$ and $\|h(t)\|_{H}=o\left(t^{-\frac{3}{4}}\right)$, then there exist $T>0$ and a curve $u(t)$ such that

- $u:[0, T] \rightarrow H$ is continuous and $u(0)=u_{0}$;

- $A^{\frac{1}{2}} u(t)$ is continuous in $H$ for $t \in(0, T]$, and $\lim _{t \rightarrow 0^{+}} t^{\frac{1}{4}}\left\|A^{\frac{1}{2}} u(t)\right\|_{H}=0$;

- $A^{\frac{3}{4}} u(t)$ is continuous in $H$ for $t \in(0, T]$, and $\lim _{t \rightarrow 0^{+}} t^{\frac{1}{2}}\left\|A^{\frac{3}{4}} u(t)\right\|_{H}=0$;

- $u(t)$ satisfies the integral equation (7) for $t \in[0, T]$;

- if $h$ is locally Hölder continuous in $(0, T]$, then $u:(0, T] \rightarrow H$ is continuously differentiable and satisfies (21); and

- the solution found is unique in the class of functions satisfying the first four properties above.

We will show that this result can be easily obtained (in an even more general form) from the results in the previous section. Moreover we will obtain uniqueness of local solutions for this problem in a larger class of functions.

The operator $A$ has an associated scale of fractional power spaces $E^{\alpha}, \alpha \in \mathbb{R}$, which satisfy $E^{0}=H$ and $E^{\alpha} \hookrightarrow H^{2 \alpha}\left(\Omega, \mathbb{R}^{N}\right), \alpha \geq 0$. From this and the continuity of the projection $P: L^{p}\left(\Omega, \mathbb{R}^{N}\right) \rightarrow L_{\sigma}^{p}\left(\Omega, \mathbb{R}^{N}\right), 1<p<\infty$, we obtain the following embeddings:

$$
\begin{array}{ll}
E^{\alpha} \hookrightarrow L_{\sigma}^{r}\left(\Omega, \mathbb{R}^{N}\right) r \leq \frac{2 N}{N-4 \alpha}, & 0 \leq \alpha<\frac{N}{4}, \\
E^{\alpha} \hookleftarrow L_{\sigma}^{s}\left(\Omega, \mathbb{R}^{N}\right) s \geq \frac{2 N}{N-4 \alpha}, & -\frac{N}{4}<\alpha \leq 0,
\end{array}
$$

where $L_{\sigma}^{r}$ is the closure of $\left\{u \in C^{2}\left(\Omega, \mathbb{R}^{N}\right): \operatorname{div} u=0, u \cdot n=0\right\}$ in $L^{r}\left(\Omega, \mathbb{R}^{N}\right)$ (see $[\mathrm{vW}]$ ). The realization of $A$ in $E^{\alpha}$ (denoted by $A_{\alpha}$ ) is an isometry from $E^{\alpha+1}$ into $E^{\alpha}$, and $A_{\alpha}: D\left(A_{\alpha}\right)=E^{\alpha+1} \subset E^{\alpha} \rightarrow E^{\alpha}$ is a sectorial operator. Furthermore, $D\left(A_{\alpha}^{\beta}\right)=E^{\alpha+\beta}$.

Denote $X^{\alpha}:=E^{\alpha-\frac{3}{4}}, \alpha \in \mathbb{R}$, and let $A: X^{1} \subset X^{0} \rightarrow X^{0}$ be the operator $A_{-\frac{3}{4}}$.

Lemma 6. For $N=3$, the nonlinearity $f$ in (21) is an $\epsilon$-regular map relative to $\left(X^{1}, X^{0}\right)$, for $\frac{1}{4} \leq \epsilon \leq \frac{3}{8}$. In this case $\rho=2$ and $\gamma(\epsilon)=2 \epsilon$. Therefore the nonlinearity is an ultra-critical map relative to $\left(X^{1}, X^{0}\right)$. 
Proof. If $\frac{1}{4} \leq \epsilon \leq \frac{3}{8}$, then

$$
L_{\sigma}^{\frac{6}{6-8 \epsilon}} \hookrightarrow X^{2 \epsilon}, \quad X^{1+\epsilon} \hookrightarrow H^{1 / 2+2 \epsilon} \hookrightarrow \mathbb{H}_{\frac{3}{2-2 \epsilon}}^{1} \hookrightarrow L^{\frac{3}{1-2 \epsilon}} .
$$

From this we have

$$
\begin{aligned}
\|P(u \cdot \nabla) u\|_{X^{2 \epsilon}} & \leq c\|P(u \cdot \nabla) u\|_{L_{\sigma}^{\frac{6}{6-8 \epsilon}}} \leq c\|(u \cdot \nabla) u\|_{L^{\frac{6}{6-8 \epsilon}}} \\
& \leq c\|u\|_{L^{\frac{6}{6-8 \epsilon}} r}\|\nabla u\|_{L^{\frac{6}{6-8 \epsilon}}} .
\end{aligned}
$$

Choosing $r=\frac{3-4 \epsilon}{1-2 \epsilon}$ and $r^{\prime}=\frac{3-4 \epsilon}{2-2 \epsilon}$ we get

$$
\|P(u \cdot \nabla) u\|_{X^{2 \epsilon}} \leq c\|u\|_{L^{\frac{6}{2-4 \epsilon}}}\|\nabla u\|_{L^{\frac{3}{2-2 \epsilon}}} \leq c\|u\|_{X^{1+\epsilon}}^{2} .
$$

Similarly we obtain

$$
\|P(u \cdot \nabla) u-P(v \cdot \nabla) v\|_{X^{2 \epsilon}} \leq c\|u-v\|_{X^{1+\epsilon}}\left(1+\|u\|_{X^{1+\epsilon}}+\|v\|_{X^{1+\epsilon}}\right) .
$$

This concludes the proof of the lemma.

It is clear from the previous lemma that the following holds.

Lemma 7. If the forcing term $h$ goes from $(0, T]$ to $X^{\gamma(\epsilon)}$, for some $\epsilon \in\left[\frac{1}{4}, \frac{3}{8}\right]$, and satisfies $\|h(t)\|_{X \gamma(\epsilon)} \leq \nu(t) t^{-2 \epsilon}$, where $0 \leq \nu(t) \leq \delta, \nu(t) \rightarrow 0$ as $t \rightarrow 0^{+}$, then $f(u)+h(t) \in \mathcal{F}(\nu(\cdot))$.

From Theorem 1 and the above lemmas we have that

Theorem 2. If there exists an $\epsilon \in\left[\frac{1}{4}, \frac{3}{8}\right]$ such that $h:(0, T] \rightarrow X^{2 \epsilon}$ and $t^{2 \epsilon}\|h(t)\|_{X^{\gamma(\epsilon)}}=\mathrm{o}(1)$; then, for any $u_{0} \in X^{1}$, the problem (21) has a unique $\epsilon-$ regular solution starting in $u_{0}$. Moreover this solution satisfies $t^{\theta}\left\|u\left(t, u_{0}\right)\right\|_{X^{1+\theta}} \rightarrow$ $0, \forall 0<\theta<\gamma(\epsilon)=2 \epsilon$.

Corollary 4 (Fujita \& Kato). If $h:(0, T] \rightarrow X^{\frac{3}{4}}=H$ and $t^{\frac{3}{4}}\|h(t)\|_{H}=\mathrm{o}(1)$, then, for any $u_{0} \in X^{1}=D\left(A^{\frac{1}{4}}\right)$, the problem (21) has a unique $\frac{3}{8}-$ regular solution starting in $u_{0}$. Moreover this solution satisfies $t^{\theta}\left\|u\left(t, u_{0}\right)\right\|_{X^{1+\theta}} \rightarrow 0, \forall 0<\theta<\frac{3}{4}$. In particular, for $\theta=\frac{1}{4}$ and $\theta=\frac{1}{2}$ we have

$$
\begin{aligned}
& \lim _{t \rightarrow 0^{+}} t^{\frac{1}{4}}\left\|A^{\frac{1}{2}} u(t)\right\|_{H}=0, \\
& \lim _{t \rightarrow 0^{+}} t^{\frac{1}{2}}\left\|A^{\frac{3}{4}} u(t)\right\|_{H}=0 .
\end{aligned}
$$

3.2. Heat Equations: $L^{q}$ Theory. There is a series of very interesting papers $[\mathrm{W} 1, \mathrm{~W} 2, \mathrm{BC}]$ that study in the spaces $L^{q}(\Omega)$ the model equation

$$
\begin{gathered}
u_{t}=\Delta u+u|u|^{\rho-1}, \quad x \in \Omega, \\
u=0, \quad x \in \partial \Omega,
\end{gathered}
$$

where $\Omega \subset \mathbb{R}^{N}$ is a bounded smooth domain. The aim is to establish for each value of $q$ the largest value of $\rho$ for which one may have existence and (maybe) uniqueness of solutions for (24).

In this section we show that most of the results in $[\mathrm{W} 1, \mathrm{~W} 2, \mathrm{BC}]$ can be easily obtained from the results in Section 2. The basic results obtained in [W1, W2, BC] are the following. 
Theorem 3 (Brezis \& Cazenáve). Assume that $q>N \frac{(\rho-1)}{2}$ and $q \geq 1$ (resp. $q=$ $\left.N \frac{(\rho-1)}{2}\right)$ and $\left.q>1\right), N \geq 1$. Given any $u_{0} \in L^{q}(\Omega)$, there exist a time $T=$ $T\left(u_{0}\right)>0$ and a unique function $u \in C\left([0, T], L^{q}(\Omega)\right)$ with $u(0)=u_{0}$ which is a classical solution of (24) on $(0, T) \times \bar{\Omega}$ in the sense that $u$ is $C^{1}$ in $t \in(0, t)$ and $C^{2}$ in $x \in \bar{\Omega}$. Moreover, we have:

- for all $t \in(0, T]$

$$
\|u(t)-v(t)\|_{L^{q}(\Omega)}+t^{\frac{N}{2 q}}\|u(t)-v(t)\|_{L^{\infty}(\Omega)} \leq C\left\|u_{0}-v_{0}\right\|_{L^{q}(\Omega)},
$$

where $T=\min \left\{T\left(u_{0}\right), T\left(v_{0}\right)\right\}$ and $C$ can be estimated in terms of $\left\|u_{0}\right\|_{L^{q}(\Omega)}$ and $\left\|v_{0}\right\|_{L^{q}(\Omega)} ;$ and

- $\lim _{t \rightarrow 0^{+}} t^{\frac{N}{2 q}}\|u(t)\|_{L^{\infty}(\Omega)}=0$.

Furthermore, the time $T$ can be chosen uniformly in compact subsets of $L^{q}(\Omega)$.

The operator $L=\Delta$ with Dirichlet boundary conditions in a bounded and smooth domain $\Omega$ can be seen as an unbounded operator in $E_{q}^{0}=L^{q}(\Omega)$, for $1<q<\infty$, with domain $E_{q}^{1}=W^{2, q}(\Omega) \cap W_{0}^{1, q}(\Omega)$. The scale of fractional powers spaces $\left\{E_{q}^{\alpha}\right\}_{\alpha \in \mathbb{R}}$ associated to $L$ satisfy

$$
\begin{aligned}
& E_{q}^{\alpha} \hookrightarrow H_{q}^{2 \alpha}(\Omega), \quad \alpha \geq 0, \quad 1<q<\infty, \\
& E_{q}^{-\alpha}=\left(E_{q^{\prime}}^{\alpha}\right)^{\prime}, \quad \alpha \geq 0, \quad 1<q<\infty, \quad q^{\prime}=\frac{q}{q-1}
\end{aligned}
$$

(see [AM2]). Therefore, from (19) and standard duality arguments, we get

$$
\begin{array}{ll}
E_{q}^{\alpha} \hookrightarrow L^{r}(\Omega) & \text { for } r \leq \frac{N q}{N-2 \alpha q}, \quad 0 \leq \alpha<\frac{N}{2 q}, \\
E_{q}^{0}=L^{q}(\Omega), & \\
E_{q}^{\alpha} \hookleftarrow L^{s}(\Omega) & \text { for } s \geq \frac{N q}{N-2 \alpha q}, \quad-\frac{N}{2 q^{\prime}}<\alpha \leq 0 .
\end{array}
$$

Moreover, the realization of $L$ in $E_{q}^{\alpha}$ (denoted by $L_{\alpha}$ ) is an isometry from $E_{q}^{\alpha+1}$ into $E^{\alpha}$, and $L_{\alpha}: D\left(L_{\alpha}\right)=E_{q}^{\alpha+1} \subset E_{q}^{\alpha} \rightarrow E_{q}^{\alpha}$ is a sectorial operator. Furthermore, $D\left(L_{\alpha}^{\beta}\right)=E_{q}^{\alpha+\beta}$.

Denote $X_{q}^{\alpha}:=E_{q}^{\alpha-1}, \alpha \in \mathbb{R}$, and let $A_{q}: X_{q}^{1} \subset X_{q}^{0} \rightarrow X_{q}^{0}$ be the operator $L_{-1}$. The fractional power spaces associated to $A_{q}$ satisfy

$$
\left.\begin{array}{ll}
X_{q}^{\alpha} \hookrightarrow L^{r}(\Omega) \quad \text { for } r \leq \frac{N q}{N+2 q-2 \alpha q}, & 1 \leq \alpha<1+\frac{N}{2 q}, \\
X_{q}^{1}=L^{q}(\Omega), & \\
X_{q}^{\alpha} \hookleftarrow L^{s}(\Omega) \quad \text { for } s \geq \frac{N q}{N+2 q-2 \alpha q}, & 1-\frac{N}{2 q^{\prime}}<\alpha \leq 1,
\end{array}\right\}
$$

with continuous embeddings.

If we consider $f: \mathbb{R} \rightarrow \mathbb{R}$ given by $f(u)=u|u|^{\rho-1}$, or in general $f$ satisfying $|f(u)-f(v)| \leq c|u-v|\left(|u|^{\rho-1}+|v|^{\rho-1}+1\right)$, we have the following.

Lemma 8 (Critical Nonlinearities). If $1<q<\infty$ and $q=N(\rho-1) / 2$, then :

- If $q>\frac{N}{N-2}$, then $f$ is an $\epsilon$-regular map relative to $\left(X_{q}^{1}, X_{q}^{0}\right)$ for $0=\epsilon_{0}(q) \leq$ $\epsilon<\frac{N}{N+2 q}$ and $\gamma(\epsilon)=\rho \epsilon$. Therefore $f$ is a critical map.

- If $q=\frac{N}{N-2}$, then $f$ is an $\epsilon$-regular map relative to $\left(X_{q}^{1}, X_{q}^{0}\right)$ for $0=\epsilon_{0}(q)<$ $\epsilon<\frac{N}{N+2 q}$ and $\gamma(\epsilon)=\rho \epsilon$. Therefore, $f$ is a double-critical map. 
- If $1<q<\frac{N}{N-2}$, then $f$ is an $\epsilon$-regular map relative to $\left(X_{q}^{1}, X_{q}^{0}\right)$ for $0<$ $\epsilon_{0}(q)<\epsilon<\frac{N}{N+2 q}$ for $\epsilon_{0}(q)=\frac{N}{N+2 q}\left(1-\frac{N}{2}\left(1-\frac{1}{q}\right)\right)>0$ and $\gamma(\epsilon)=\rho \epsilon$. Therefore, $f$ is an ultra-critical map.

Proof. Just use the embeddings (26).

Now it is clear that, applying Theorem 1 , for each $u_{0} \in L^{q}(\Omega)$ we have the existence of a unique $\epsilon$-regular solution of the above problem, starting at $u_{0}$, for any $\epsilon \in\left(\epsilon_{0}(q), \frac{N}{N+2 q}\right)$. Moreover, for any $0<\theta<\gamma\left(\frac{N}{N+2 q}\right)=1$, this solution satisfies

$$
\begin{gathered}
t^{\theta}\|u(t)\|_{X^{1+\theta}} \rightarrow 0, \quad \text { as } t \rightarrow 0^{+}, \\
t^{\theta}\left\|u\left(t, u_{0}\right)-u\left(t, v_{0}\right)\right\|_{X^{1+\theta}} \leq C\left\|u_{0}-v_{0}\right\|_{L^{q}}, \quad 0<t<\tau\left(u_{0}, v_{0}\right) .
\end{gathered}
$$

In particular, since $X_{q}^{1}=L^{q}(\Omega)$, we get that $X^{1+\theta} \hookrightarrow W^{2 \theta, q} \hookrightarrow L^{p}$ for $p=\frac{N q}{N-2 \theta q}>$ $q$, if $\theta>0$. Therefore, there exists a $p_{0}>q$ such that

$$
\begin{gathered}
t^{\frac{N}{2}\left(\frac{1}{q}-\frac{1}{p_{0}}\right)}\|u(t)\|_{L^{p_{0}}} \stackrel{t \rightarrow 0^{+}}{\longrightarrow} 0, \\
t^{\frac{N}{2}\left(\frac{1}{q}-\frac{1}{p_{0}}\right)}\left\|u\left(t, u_{0}\right)-u\left(t, v_{0}\right)\right\|_{L^{p_{0}}} \leq C\left\|u_{0}-v_{0}\right\|_{L^{q}}, \quad 0<t<\tau\left(u_{0}, v_{0}\right) .
\end{gathered}
$$

Now it is possible to apply a bootstrap argument to show that in fact

$$
\begin{gathered}
t^{\frac{N}{2 q}}\|u(t)\|_{L^{\infty}} \rightarrow 0 \text { as } t \rightarrow 0^{+}, \\
t^{\frac{N}{2 q}}\left\|u\left(t, u_{0}\right)-u\left(t, v_{0}\right)\right\|_{L^{\infty}} \leq C\left\|u_{0}-v_{0}\right\|_{L^{q}}, \quad 0<t<\tau\left(u_{0}, v_{0}\right) .
\end{gathered}
$$

For this let us establish the following lemma:

Lemma 9. There exists $\eta>0$ small enough so that if $p \geq p_{0}$, then

$$
\frac{N}{2}\left(\frac{\rho}{p}-\frac{1}{p+\eta}\right)<1, \quad \forall p \geq p_{0} .
$$

Proof. Note that

$$
\frac{N}{2}\left(\frac{\rho}{p}-\frac{1}{p+\eta}\right)=\frac{q}{p}+\frac{N}{2}\left(\frac{1}{p}-\frac{1}{p+\eta}\right) \leq \frac{q}{p_{0}}+\frac{N}{2}\left(\frac{1}{p}-\frac{1}{p+\eta}\right) .
$$

Choose $\eta$ so small that $\frac{N}{2}\left(\frac{1}{p}-\frac{1}{p+\eta}\right)<1-\frac{q}{p_{0}}$, for all $p \geq p_{0}$. This proves the result.

The next step is to use an induction argument as follows:

Lemma 10. Let $\eta$ and $p_{0}$ be as above and define the sequence $p_{n}$ by $p_{n+1}=p_{n}+\eta$. If

$$
t^{\frac{N}{2}\left(\frac{1}{q}-\frac{1}{p_{n}}\right)}\|u(t)\|_{L^{p_{n}}(\Omega)} \rightarrow 0 \quad \text { as } t \rightarrow 0^{+},
$$

then

$$
t^{\frac{N}{2}\left(\frac{1}{q}-\frac{1}{p}\right)}\|u(t)\|_{L^{p}(\Omega)} \rightarrow 0 \quad \text { as } t \rightarrow 0^{+} \forall p \in\left[p_{n}, p_{n+1}\right] .
$$

Proof. Using the expression

$$
u(t)=e^{-\Delta \frac{t}{2}} u\left(\frac{t}{2}\right)+\int_{\frac{t}{2}}^{t} e^{-\Delta(t-s)} f(u(s)) d s
$$


we have

$$
\begin{aligned}
& t^{\frac{N}{2}\left(\frac{1}{q}-\frac{1}{p}\right)}\|u\|_{L^{p}} \\
& \leq t^{\frac{N}{2}\left(\frac{1}{q}-\frac{1}{p}\right)}\left\|e^{-\Delta \frac{t}{2}} u\left(\frac{t}{2}\right)\right\|_{L^{p}}+t^{\frac{N}{2}\left(\frac{1}{q}-\frac{1}{p}\right)} \int_{\frac{t}{2}}^{t}(t-s)^{-\frac{N}{2}\left(\frac{\rho}{p_{n}}-\frac{1}{p}\right)}\|f(u)\|_{L^{\frac{p_{n}}{\rho}}} \\
& \leq t^{\frac{N}{2}\left(\frac{1}{q}-\frac{1}{p}\right)}\left\|e^{-\Delta \frac{t}{2}} u\left(\frac{t}{2}\right)\right\|_{L^{p}}+c t^{\frac{N}{2}\left(\frac{1}{q}-\frac{1}{p}\right)} \int_{\frac{t}{2}}^{t}(t-s)^{-\frac{N}{2}\left(\frac{\rho}{p_{n}}-\frac{1}{p}\right)}\left(1+\|u\|_{L^{p_{n}}}^{\rho}\right) \\
& \leq t^{\frac{N}{2}\left(\frac{1}{q}-\frac{1}{p}\right)}\left(\frac{t}{2}\right)^{\frac{N}{2}\left(\frac{1}{p}-\frac{1}{p_{n}}\right)}\left\|u\left(\frac{t}{2}\right)\right\|_{L^{p_{n}}}+c t^{\frac{N}{2}\left(\frac{1}{q}-\frac{1}{p}\right)} \int_{\frac{t}{2}}^{t}(t-s)^{-\frac{N}{2}\left(\frac{\rho}{p_{n}}-\frac{1}{p}\right)} d s \\
& \quad+c t^{\frac{N}{2}\left(\frac{1}{q}-\frac{1}{p}\right)} \int_{\frac{t}{2}}^{t}(t-s)^{-\frac{N}{2}\left(\frac{\rho}{p_{n}}-\frac{1}{p}\right)} s^{-\frac{N}{2}\left(\frac{1}{q}-\frac{1}{p_{n}}\right) \rho} d s \sup _{0 \leq s \leq t}\left(s^{\frac{N}{2}\left(\frac{1}{q}-\frac{1}{p_{n}}\right)}\|u(s)\|_{\left.L^{p_{n}}(\Omega)\right)^{\rho}}\right. \\
& \leq c \sup _{0<s<t}\left(s^{\frac{N}{2}\left(\frac{1}{q}-\frac{1}{p_{n}}\right)}\|u(s)\|_{L^{p_{n}}}\right)+c t^{\frac{N \rho}{2}\left(\frac{1}{q}-\frac{1}{p_{n}}\right)} \int_{\frac{1}{2}}^{1}(1-s)^{-\frac{N}{2}\left(\frac{\rho}{p_{n}}-\frac{1}{p}\right)} d s \\
& \quad+c \int_{\frac{1}{2}}^{1}(1-s)^{-\frac{N}{2}\left(\frac{\rho}{p_{n}}-\frac{1}{p}\right)} s^{-\frac{N}{2}\left(\frac{1}{q}-\frac{1}{p_{n}}\right) \rho} d s \sup _{0 \leq s \leq t}\left(s^{\frac{N}{2}\left(\frac{1}{q}-\frac{1}{p_{n}}\right)}\|u(s)\|_{\left.L^{p_{n}}(\Omega)\right)^{\rho}} .\right.
\end{aligned}
$$

Notice that from the previous lemma we have $\frac{N}{2}\left(\frac{\rho}{p_{n}}-\frac{1}{p}\right)<1$, and therefore all the integrals are well defined. Hence $\sup \left(s^{\frac{N}{2}\left(\frac{1}{q}-\frac{1}{p_{n}}\right)}\|u(s)\|_{L^{p_{n}}}\right) \rightarrow 0$ as $t \rightarrow 0^{+}$. Also, since $p_{n}>q$ we have $t^{\frac{N \rho}{2}\left(\frac{1}{q}-\frac{1}{p_{n}}\right)} \rightarrow 0$ as $t \rightarrow 0$.

This concludes the proof.

Note that from (27) and the previous lemma we get that

$$
t^{\frac{N}{2}\left(\frac{1}{q}-\frac{1}{p}\right)}\|u\|_{L^{p}} \rightarrow 0, \quad \forall p \geq p_{0} .
$$

In order to estimate the $L^{\infty}$ norm we choose $p>\frac{N \rho}{2}$, and with a similar argument as above we can show that

$$
\begin{aligned}
& t^{\frac{N}{2 q}}\|u\|_{L^{\infty}} \leq t^{\frac{N}{2 q}}\left\|e^{-\Delta \frac{t}{2}} u\left(\frac{t}{2}\right)\right\|_{L^{\infty}}+t^{\frac{N}{2 q}} \int_{\frac{t}{2}}^{t}(t-s)^{-\frac{N \rho}{2 p}}\|f(u)\|_{L^{p / \rho}} \\
& \leq t^{\frac{N}{2 q}}(t / 2)^{-\frac{N}{2 p}}\left\|u\left(\frac{t}{2}\right)\right\|_{L^{p}}+t^{\frac{N}{2 q}} \int_{\frac{t}{2}}^{t}(t-s)^{-\frac{N \rho}{2 p}} c\left(1+\|u\|_{L^{p}}^{\rho}\right) d s \\
& \leq t^{\frac{N}{2}\left(\frac{1}{q}-\frac{1}{p}\right)}\|u(t)\|_{L^{p}}+c t^{\frac{N}{2 q}} \int_{\frac{t}{2}}^{t}(t-s)^{-\frac{N \rho}{2 p}} d s \\
& \quad+c t^{\frac{N}{2 q}} \int_{\frac{t}{2}}^{t}(t-s)^{-\frac{N \rho}{2 p}} s^{-\rho \frac{N}{2}\left(\frac{1}{q}-\frac{1}{p}\right)} d s \sup _{0<s<t}\left[s^{\frac{N}{2}\left(\frac{1}{q}-\frac{1}{p}\right)}\|u(s)\|_{L^{p}}\right]^{\rho} \rightarrow 0
\end{aligned}
$$

as $t \rightarrow 0$, because of reasons similar to the argument above.

This shows the first statement of (28).

For the second one the analysis is similar. Starting with the second statement of (27) and with a similar bootstrap argument as above, we can obtain the desired estimate.

Now it is not difficult to obtain certain estimates of the behavior of the $C^{\alpha}$ norm of the solutions as $t \rightarrow 0^{+}$. We have 
Proposition 2. If $q=\frac{N(\rho-1)}{2}>1$ and $|f(u)-f(v)| \leq c|u-v|\left(|u|^{\rho-1}+|v|^{\rho-1}+1\right)$, then, for any $\eta<1$, we have

$$
\begin{gathered}
t^{1+\frac{N}{2 q}}\|u(t)\|_{C^{1, \eta}} \rightarrow 0 \quad \text { as } t \rightarrow 0, \\
t^{1+\frac{N}{2 q}}\|u(t)-v(t)\|_{C^{1, \eta}} \leq C\left\|u_{0}-v_{0}\right\|_{L^{q}} .
\end{gathered}
$$

Proof. Let us start with the following result.

Lemma 11. With the hypothesis above, if $p$ is large enough, we have

$$
f: X_{p}^{1}=L^{p} \rightarrow L^{\frac{p}{\rho}} \hookrightarrow X_{p}^{\alpha},
$$

with $\alpha=\frac{N}{2 p}(\rho-1)$, and

$$
\|f(u)-f(v)\|_{X_{p}^{\alpha}} \leq c\|u-v\|_{X_{p}^{1}}\left(\|u\|_{X_{p}^{1}}^{\rho-1}+\|v\|_{X_{p}^{1}}^{\rho-1}+1\right) .
$$

That is, $f$ is a subcritical map relative to the pair $\left(X_{p}^{1}, X_{p}^{0}\right)$.

Proof. We use (26) again.

Proof of the proposition. Let $\beta<1$ and choose $p$ large enough so that $\alpha=$ $\frac{N}{2 p}(\rho-1)<1-\beta$. Then

$$
\begin{aligned}
& t^{\beta+\frac{N}{2}\left(\frac{1}{q}-\frac{1}{p}\right)}\|u(t)\|_{X_{p}^{1+\beta}} \\
& \leq t^{\beta+\frac{N}{2}\left(\frac{1}{q}-\frac{1}{p}\right)}\left\|e^{-\Delta \frac{t}{2}} u\left(\frac{t}{2}\right)\right\|_{X_{p}^{1+\beta}}+t^{\beta+\frac{N}{2}\left(\frac{1}{q}-\frac{1}{p}\right)} \int_{\frac{t}{2}}^{t} e^{-\Delta(t-s)} f(u(s)) d s \|_{X_{p}^{1+\beta}} \\
& \leq t^{\beta+\frac{N}{2}\left(\frac{1}{q}-\frac{1}{p}\right)}\left(\frac{t}{2}\right)^{-\beta}\left\|u\left(\frac{t}{2}\right)\right\|_{X_{p}^{1}} \\
&+t^{\beta+\frac{N}{2}\left(\frac{1}{q}-\frac{1}{p}\right)} \int_{\frac{t}{2}}^{t}(t-s)^{-(\beta+\alpha)} c\left(1+\|u(s)\|_{X_{p}^{1}}^{\rho}\right) d s \\
& \leq c t^{\frac{N}{2}\left(\frac{1}{q}-\frac{1}{p}\right)}\left\|u\left(\frac{t}{2}\right)\right\|_{X_{p}^{1}}+c t^{\beta+\frac{N}{2}\left(\frac{1}{q}-\frac{1}{p}\right)} \int_{\frac{t}{2}}^{t}(t-s)^{-(\beta+\alpha)} d s \\
&+c t^{\beta+\frac{N}{2}\left(\frac{1}{q}-\frac{1}{p}\right)} \int_{\frac{t}{2}}^{t}(t-s)^{-(\beta+\alpha)} s^{-\frac{N}{2}\left(\frac{1}{q}-\frac{1}{p}\right) \rho} d s \sup _{0<s<t}\left(s^{\frac{N}{2}\left(\frac{1}{q}-\frac{1}{p}\right)}\|u(s)\|_{X_{p}^{1}}\right)^{\rho} \\
& \stackrel{t \rightarrow 0^{+}}{\longrightarrow} 0
\end{aligned}
$$

for reasons similar to the arguments above.

The proof of the first statement of (30) follows from the above result and the fact that for any $\eta<1$ we can choose a $\beta<1$ and a $p$ large enough, with the property that $X_{p}^{1+\beta} \hookrightarrow H_{p}^{2 \beta} \hookrightarrow C^{1, \eta}$ (see statement (19)).

For the second part of statement (30) the argument is similar. This concludes the proof of the proposition.

So far we have treated the case where $q=\frac{N}{2}(\rho-1)$, that is, critical cases. In principle, the treatment of the cases $q>\frac{N}{2}(\rho-1)$ is simpler. It can be seen that:

- If $\frac{N}{N-2} \leq q$ and $\frac{N}{2}(\rho-1)<q$, then $f$ is a subcritical map relative to $\left(X_{q}^{1}, X_{q}^{0}\right)$.

- If $1<q<\frac{N}{N-2}$ and $\rho \leq q$, then $f$ is a subcritical map relative to $\left(X_{q}^{1}, X_{q}^{0}\right)$.

- If $1<q<\frac{N}{N-2}$ and $\frac{N}{2}(\rho-1)<q<\rho$, then $f$ is an ultra-subcritical map relative to $\left(X_{q}^{1}, X_{q}^{0}\right)$. 
In order to visualize this classification we designed Figures 1,2 and 3.

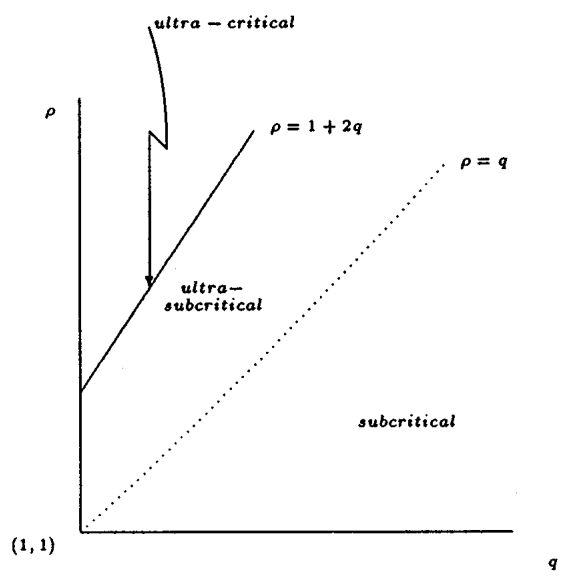

FIgURE 1. Criticality of $f$ in $L^{q}(\Omega)$ for $N=1$

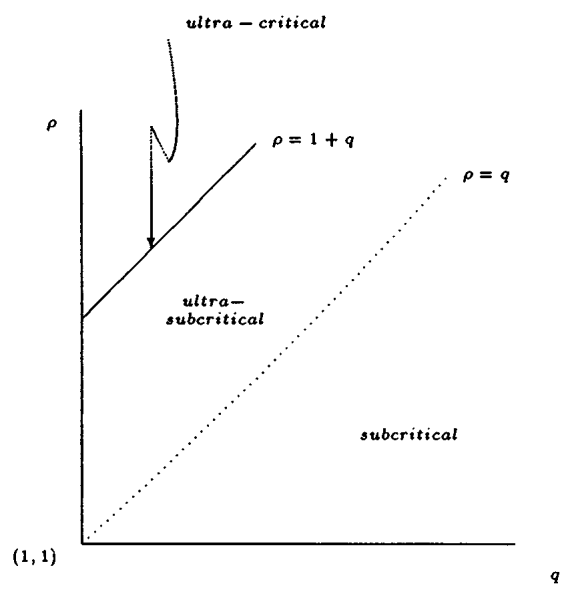

Figure 2. Criticality of $f$ in $L^{q}(\Omega)$ for $N=2$

3.3. Heat Equations: $W^{1, q}$ Theory. Once we have the results from Section 2, the $W^{1, q}$-theory for heat equations is not much different from the $L^{q}$ theory. Again, what we need is a good understanding of the fractional power spaces of the linear operator (already done in the $L^{q}$ setting), and of the $\epsilon$-regularity properties of the nonlinearities involved.

Roughly speaking, we will see that, in this case, for $1<q<N$ the critical growth exponents are $\rho=\frac{N+q}{N-q}$, that for $q>N$ there is no critical exponent due to the embedding of $W^{1, q}(\Omega) \hookrightarrow C(\bar{\Omega})$, and that for $q=N$ the critical growth is larger than exponential and is established by Trudinger's inequality (see, [Tr, Mo]).

Let $E_{q}^{\alpha}, \alpha \in \mathbb{R}, 1<q<\infty$, be, as in the previous section, the fractional power spaces of the Laplace operator with Dirichlet boundary condition in $L^{q}(\Omega)$. Denote 


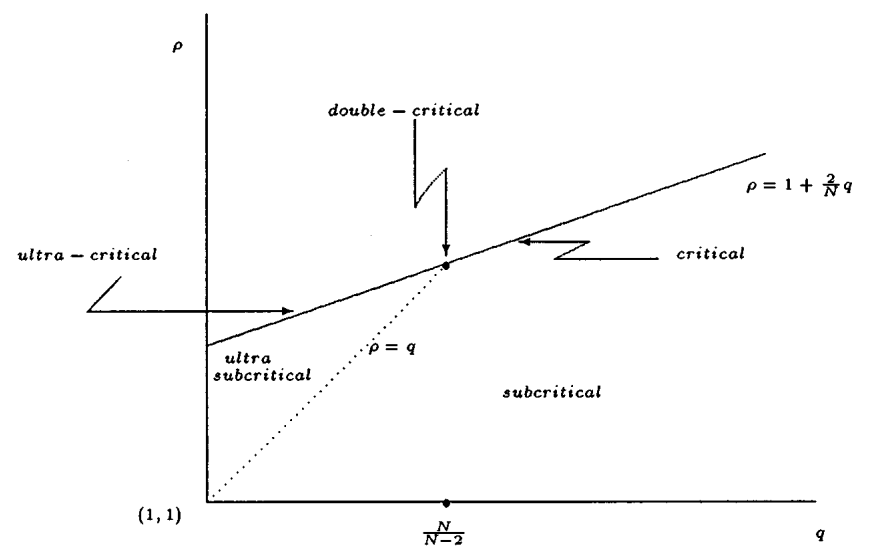

Figure 3. Criticality of $f$ in $L^{q}(\Omega)$ for $N=3$

$X_{q}^{\alpha}:=E_{q}^{\alpha-\frac{1}{2}}, \alpha \in \mathbb{R}$, and by $A_{q}: X_{q}^{1} \subset X_{q}^{0} \rightarrow X_{q}^{0}$ the operator $L_{-\frac{1}{2}}$. Moreover the fractional power spaces $X_{q}^{\alpha}$ associated to $A_{q}$ satisfy

$$
\begin{aligned}
& X_{q}^{\alpha} \hookrightarrow L^{r}(\Omega), \quad \text { for } r \leq \frac{N q}{N+q-2 \alpha q}, \quad \frac{1}{2} \leq \alpha<\frac{1}{2}+\frac{N}{2 q}, \\
& X_{q}^{\frac{1}{2}}=L^{q}(\Omega), \\
& X_{q}^{\alpha} \hookleftarrow L^{s}(\Omega), \quad \text { for } s \geq \frac{N q}{N+q-2 \alpha q}, \quad \frac{1}{2}-\frac{N}{2 q^{\prime}}<\alpha \leq \frac{1}{2} .
\end{aligned}
$$

Assume that $f: \mathbb{R} \rightarrow \mathbb{R}$ is a $C^{1}$ map. In the $W^{1, q}$ theory we will need the following two growth conditions:

$$
\begin{gathered}
|f(u)-f(v)| \leq c|u-v|\left(1+|u|^{\rho-1}+|v|^{\rho-1}\right), \quad u \in \mathbb{R}, \\
\lim _{|u| \rightarrow \infty} \frac{\left|f^{\prime}(u)\right|}{e^{\eta|u|^{N-1}}}=0, \quad \forall \eta>0, \quad u \in \mathbb{R} .
\end{gathered}
$$

The $\epsilon$-regularity properties of the map $f$ are given by the following lemma.

Proposition 3. The nonlinearity $f$ can be classified as follows:

- If $q>N$, then any $f \in C^{1}$ is a subcritical map relative to $\left(X_{q}^{1}, X_{q}^{0}\right)$.

- If $q=N$, then any $f \in C^{1}$ which satisfies (33) is a subcritical map relative to $\left(X_{q}^{1}, X_{q}^{0}\right)$.

- If $\frac{N}{N-1}<q<N$ and $f \in C^{1}$ satisfy (32), then:

1. If $\rho=\frac{N+q}{N-q}$, $f$ is an $\epsilon$-regular map relative $\left(X_{q}^{1}, X_{q}^{0}\right)$ for $0 \leq \epsilon<\frac{1}{2 \rho}$. Therefore $f$ is a critical map.

2. If $\rho<\frac{N+q}{N-q}$, $f$ is a subcritical map relative to $\left(X_{q}^{1}, X_{q}^{0}\right)$.

- If $\frac{N}{N-1}=q$ and $f \in C^{1}$ satisfy (32), then:

1. If $\rho=\frac{N+q}{N-q}=\frac{N}{N-2}, f$ is an $\epsilon-$ regular map relative to $\left(X_{q}^{1}, X_{q}^{0}\right)$ for $0<\epsilon<\frac{1}{2 \rho}$. Therefore $f$ is a double-critical map.

2. If $\rho<\frac{N}{N-2}, f$ is a subcritical map relative to $\left(X_{q}^{1}, X_{q}^{0}\right)$.

- If $1<q<\frac{N}{N-1}$ and $f \in C^{1}$ satisfying (32), then: 
1. If $\rho=\frac{N+q}{N-q}$, $f$ is an $\epsilon$-regular map relative to $\left(X_{q}^{1}, X_{q}^{0}\right)$ for $0<\epsilon_{0}=$ $\frac{N-q}{2 q}-\frac{N}{2 \rho}<\epsilon<\epsilon_{1}=\frac{N-q}{2 q}-\frac{N}{2 q \rho}$, with $\gamma(\epsilon)=\rho \epsilon$. Therefore $f$ is an ultra-critical map.

2. If $\frac{N q}{N-q}<\rho<\frac{N+q}{N-q}$, $f$ is an $\epsilon-$ regular map relative to $\left(X_{q}^{1}, X_{q}^{0}\right)$ for $0<\epsilon_{0}=\frac{N-q}{2 q}-\frac{N}{2 \rho}<\epsilon<\epsilon_{1}=\frac{N-q}{2 q}-\frac{N}{2 q \rho}$, with $\gamma(\epsilon)>\rho \epsilon$. Therefore $f$ is an ultra-subcritical map.

3. If $1<\rho \leq \frac{N q}{N-q}$, $f$ is a subcritical map relative to $\left(X_{q}^{1}, X_{q}^{0}\right)$.

In order to visualize this classification we include Figures 4,5 and 6 , which are similar to the figures from the $L^{q}$ theory.

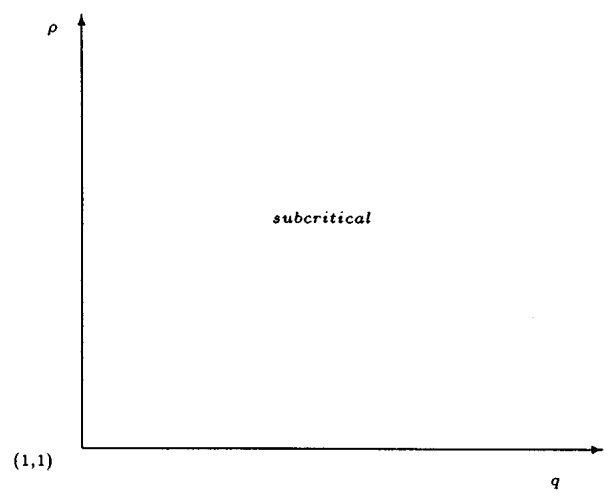

Figure 4. Criticality of $f$ in $W^{1, q}(\Omega)$ for $N=1$

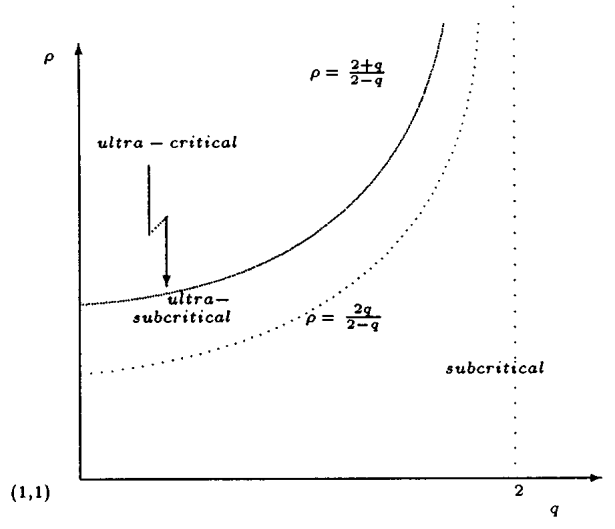

Figure 5. Criticality of $f$ in $W^{1, q}(\Omega)$ for $N=2$ 


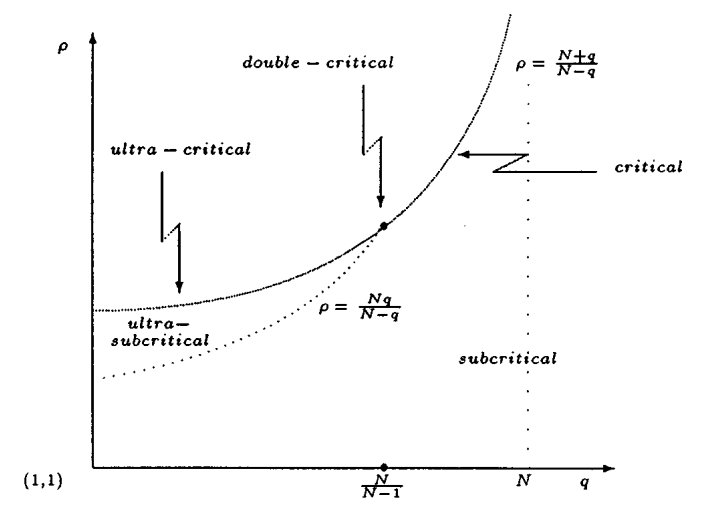

Figure 6 . Criticality of $f$ in $W^{1, q}(\Omega)$ for $N=3$

Proof. All the proofs follow from the embeddings (19) and (31) except for the case $q=N$, for which the proof is based in the following lemma due to N. Trudinger (see $[\mathrm{Tr}, \mathrm{Mo}])$.

Lemma 12. Given $p \geq 1$ and $\sigma \leq \frac{1}{p} N \omega_{N-1}^{\frac{1}{N-1}}$, there exists a positive constant $K$ such that, if $u \in W^{1, N}\left(\Omega, \mathbb{R}^{n}\right),\|u\|_{W^{1, N}} \leq 1$, then

$$
\left\|e^{\sigma|u(\cdot)|^{\frac{N}{N-1}}}\right\|_{L^{p}} \leq K
$$

where $\omega_{N-1}$ is the $(N-1)$-dimensional surface of the unit sphere.

Proof. Let us prove that the function $f: X_{N}^{1} \rightarrow X_{N}^{\frac{1}{2}}$ is Lipschitz continuous in bounded subsets of $X^{1}$.

Let $r>0$, and let $u$ and $v$ be functions in $W^{1, N}$ such that $\|u\|_{W^{1, N}} \leq r$, $\|v\|_{W^{1, N}} \leq r$. Let $\eta<\frac{\sigma}{2 N r^{N-1}}$. Then, from (33), there exists $c_{\eta}>0$ such that

$$
|f(u)-f(v)|^{N} \leq c_{\eta}\left(e^{N \eta|u|^{N-1}}+e^{N \eta|v|^{N-1}}\right)|u-v|^{N},
$$

and

$$
\begin{aligned}
& \|f(\phi)-f(\psi)\|_{L^{N}(\Omega)}^{N} \leq c_{\eta} \int_{\Omega}\left[e^{N \eta|\phi(x)|^{\frac{N}{N^{-1}}}}+e^{\left.N \eta|\psi(x)|^{\frac{N}{N^{-1}}}\right]|\phi(x)-\psi(x)|^{N} d x}\right. \\
& \leq c_{\eta}\left(\int_{\Omega}\left[e^{N \eta|\phi(x)|^{\frac{N}{N-1}}}+e^{N \eta|\psi(x)|^{\frac{N}{N-1}}}\right]^{2} d x\right)^{\frac{1}{2}}\left(\int_{\Omega}|\phi(x)-\psi(x)|^{2 N} d x\right)^{\frac{1}{2}} \\
& \leq c_{\eta}\|\phi-\psi\|_{L^{2 N}(\Omega)}^{N}\left(\int_{\Omega}\left[e^{N \eta|\phi(x)|^{\frac{N}{N-1}}}+e^{N \eta|\psi(x)|^{\frac{N}{N-1}}}\right]^{2} d x\right)^{\frac{1}{2}} \\
& \leq \bar{c}_{\eta}\|\phi-\psi\|_{W^{1, N}}^{N}\left(\int_{\Omega}\left[e^{2 N \eta|\phi(x)|^{\frac{N}{N-1}}}+e^{2 N \eta|\psi(x)|^{\frac{N}{N-1}}}\right] d x\right)^{\frac{1}{2}} .
\end{aligned}
$$

The result now follows from the fact that $X_{N}^{1} \subset W^{1, N}$ with continuous embedding, $X_{N}^{\frac{1}{2}}=L^{N}$, and from the fact that

$$
\left\|e^{2 N \eta|\phi(\cdot)|^{\frac{N}{N-1}}}\right\|_{L^{1}} \leq K,
$$

which comes from Lemma 12 . 


\section{A COMMENT ON UNIQUENESS}

In Section 2 we have been able to establish the existence and uniqueness of $\epsilon$ regular solutions for the problem $\dot{x}=A x+f(x)$, where $f$ is an $\epsilon$-regular map. This $\epsilon$-regular solutions are characterized by immediate regularization properties for $t>0$. Therefore, uniqueness is established in the class of functions $C\left((0, \tau], X^{1+\epsilon}\right)$. It is natural and interesting to ask whether uniqueness can be obtained in the larger space $C\left([0, \tau], X^{1}\right)$. This is equivalent to establishing uniqueness of mild solutions (not just $\epsilon$-regular mild solutions).

In this respect there are several results in the literature that can give some insight into this problem in the abstract setting.

In $[\mathrm{NS}], \mathrm{Ni}$ and Sacks were able to give a non-uniqueness result in $C\left([0, \tau], L^{q}(\Omega)\right)$ for the heat equation (24) when $q=\frac{N}{2}(\rho-1)$ and $q=\rho$ (see also [BC]). This is exactly the case $q=\rho=\frac{N}{N-2}$, which in our classification of nonlinearities corresponds to the double critical case. In [BC] (Theorem 4), Brezis and Cazenáve were able to give a uniqueness result in the space $C\left([0, \tau], X^{1}\right)$ for the problem $(24)$ when $q=\frac{N}{2}(\rho-1)$ and $q>\rho$. In our classification of nonlinearities this corresponds to the critical case. Recently, the authors have learned of a uniqueness result for the Navier-Stokes equations in $L^{3}\left(R^{3}\right)$ by P.G. Lemarié-Rieusset (see $[\mathrm{LR}]$ ). He proved the uniqueness of solutions in the space $C\left([0, \tau), L^{3}\left(R^{3}\right)\right)$. The proof uses a very nice bound of the integral expression from the variation of constant formula in certain Besov spaces.

We tried, unsuccessfully, to prove an abstract uniqueness result in $C\left([0, \tau], X^{1}\right)$, for the critical case, but this uniqueness result seems plausible. The main difference between the critical case and the double critical case is that in the former the map $f$ is $\epsilon$-regular even for $\epsilon=0$. This means that $f$ transforms $X^{1}$ into $X^{0}$. In the double critical case the $\epsilon$-regularity properties of $f$ start for $\epsilon>0$, and therefore $f$ does not transform $X^{1}$ into $X^{0}$. Any proof of a uniqueness result for the critical case should exploit this fact.

Needless to say to prove or disprove any of the uniqueness results mentioned above will be extremely important for a full understanding of the subject.

\section{REFERENCES}

[AD] Adams, R. Sobolev Spaces, Academic Press, 1975. MR 56:9247

[AM1] H. Amann, On abstract parabolic fundamental solutions, J. Math. Soc. Japan 39, (1987), 93-116. MR 88b:34086

[AM2] H. Amann, Nonhomogeneous linear and quasilinear elliptic and parabolic boundary value problems, in: Schmeisser/Triebel: Function Spaces, Differential Operators and Nonlinear Analysis, Teubner Texte zur Mathematik, vol. 133, Teubner, 1993, pp. 9-126. MR 94m:35153

[AM3] H. Amann, Linear and Quasilinear Parabolic Problems. Abstract Linear Theory. Birkhäuser Verlag, 1995. MR 96g:34088

[BC] Brezis, H. \& Cazenáve, T., A nonlinear heat equation with singular initial data, J. Anal. Math., 68, (1996), 277-304. MR 97f:35092

[FK] Fujita, H. \& Kato, T., On the Navier-Stokes initial value problem I. Arch. Rat. Mech. Anal. 16 (1964), 269-315. MR 29:3774

[Fu] Fujiwara, D., On the asymptotic behavior of the Green operators for elliptic boundary problems and the pure imaginary powers of some second order operators. J. Math. Soc. Japan 21, (1969), 481-521. MR 41:7287

[HE] Henry, D., Geometric theory of semilinear parabolic equations, Lecture Notes in Mathematics 840, Springer-Verlag, Berlin, (1981). MR 83j:35084 
[KF] Kato. T. \& Fujita, H., On the nonstationary Navier-Stokes system. Rend. Sem. Math. Univ. Padova 32, (1962), 243-260. MR 26:495

[LR] Lemarié-Rieusset, P.G. Unicité des solutions des équations de Navier-Stokes, handwritten notes.

[Mo] Moser, J., A sharp form of an inequality by N. Trudinger, Indiana U. Math. J., 20, (1971), 1077-1092. MR 46:662

[NS] Ni, W.M. \& Sacks, P., Singular behavior in nonlinear parabolic equations. Trans. Amer. Math. Soc., 287, (1985), 657-671. MR 86i:35073

[PS] Prüss, J and Sohr, H., Imaginary powers of elliptic second order differential operators in $L^{p}$-spaces. Hiroshima Math. J., 23, (1993), 161-192. MR 94d:47051

[S] Seeley, R., Norms and domains of the complex powers $A_{B}^{z}$. American Journal of Mathematics, 93 (1971), 299-309. MR 44:4582

[Tri] Triebel, H. Interpolation Theory, Function Spaces, Differential Operators, North Holland, Amsterdam, 1978. MR 80i:46032

[Tr] Trudinger, N. S., On imbeddings into Orlicz spaces and some applications, Journal of Mathematics and Mechanics, 17, (1967), 473-483. MR 35:7121

[vW] von Wahl, W. The Equations of Navier-Stokes and Abstract Parabolic Equations, Vieweg \& Sohn, Braunschweig, (1985). MR 88a:35195

[W1] Weissler, F. B., Semilinear evolution equations in Banach spaces, J. Functional Anal., 32, (1979), 277-296. MR 80i:47091

[W2] Weissler, F. B., Local existence and nonexistence for semilinear parabolic equations in $L^{p}$, Indiana U. Math. J., 29, (1980), 79-102. MR 81e:35072

Departamento de Matemática Aplicada, Facultad de Matemáticas, Universidad ComPLUTENSE DE MADRID, 28040 MADRID, SPAIN

E-mail address: arrieta@sunma4.mat.ucm.es

Departamento de Matemática, instituto de Ciências Matemáticas de São Carlos, Universidade de São Paulo, C.P. 668, São Carlos, SP. Brazil

E-mail address: andcarva@icmsc.sc.usp.br 